\title{
Properties of Input-Consuming Derivations
}

\author{
Annalisa Bossi ${ }^{1}$, Sandro Etalle $^{2}$, Sabina Rossi ${ }^{1}$ \\ ${ }^{1}$ Dipartimento di Informatica, Università di Venezia \\ via Torino 155, 30172 Venezia, Italy \\ ${ }^{2}$ Department of Computer Science, University of Maastricht \\ P.O. Box 616, 6200 MD Maastricht, The Netherlands \\ and \\ CWI - Center for Mathematics and Computer Science, \\ P.O. Box 94079, 1090 GB Amsterdam, The Netherlands
}

October 31, 2018

\begin{abstract}
We study the properties of input-consuming derivations of moded logic programs. Input-consuming derivations can be used to model the behavior of logic programs using dynamic scheduling and employing constructs such as delay declarations.

We consider the class of nicely-moded programs and queries. We show that for these programs a weak version of the well-known switching lemma holds also for input-consuming derivations. Furthermore, we show that, under suitable conditions, there exists an algebraic characterization of termination of input-consuming derivations.
\end{abstract}

\section{Introduction}

Most of the recent logic programming languages provide the possibility of employing dynamic scheduling, i.e., a runtime mechanism determining which atoms in a query are selectable and which ones are not. In fact, dynamic scheduling has proven to be useful in a number of applications; among other things, it allows one to model coroutining, as shown in Nai92, HL94, and parallel executions, as shown in Nai88.

Let us use the following simple examples to show how dynamic scheduling can be enforced by using delay declarations and how it can prevent nontermination and unnecessary computations. Consider the program APPEND

$\operatorname{app}([], Y s, Y s)$.

$\operatorname{app}([\mathrm{H} \mid \mathrm{Xs}], \mathrm{Ys},[\mathrm{H} \mid \mathrm{Zs}]) \leftarrow \operatorname{app}(\mathrm{Xs}, \mathrm{Ys}, \mathrm{Zs})$. 
together with the query

$$
Q_{1}:=\operatorname{app}\left(\mathrm{Xs}_{\mathbf{s}},[5,6], \mathrm{Ys}\right), \operatorname{app}([1,2],[3,4], \mathrm{Xs}) .
$$

In this query, if we select and resolve the leftmost atom, we could easily have to face one of the following two problems. First, the possibility of nontermination: This is the case if we repeatedly resolve the leftmost atom against the second clause. The second problem is that of inefficiency. If, for instance, in $Q_{1}$ we resolve the leftmost atom against the first clause, we obtain the query $\operatorname{app}([1,2],[3,4],[])$. This will eventually fail, yielding to (unnecessary) backtracking. Notice that if one employs the rightmost selection rule, $Q_{1}$ would terminate with success and without backtracking. Basically, the problem when selecting app (Xs, $[5,6], Y s$ ), is that we do not know which clause we should use for resolving it, and the only practical way for getting to know this is by waiting until the outermost functor of Xs is known: If it is the empty list [ ] we know that we should use the first clause, if it is the list-constructor symbol we know that we should use the second clause, if it is something else again, we know then that the query fails. Notice that the same problems arise for the query

$$
Q_{2}:=\operatorname{app}\left([1,2],[3,4], X_{\mathbf{s}}\right), \operatorname{app}\left(\mathrm{Xs}_{\mathbf{s}},[5,6], \mathrm{Ys}\right) .
$$

if the rightmost selection rule is considered.

This shows the usefulness of a mechanism for preventing the selection of those atoms which are not sufficiently instantiated. Such a mechanism is in fact offered by most modern languages: In GHC Ued88 programs are augmented with guards in order to control the selection of atoms dynamically. Moded Flat GHC UM94 uses an extra condition on the input positions, which is extremely similar to the concept of input-consuming derivation step we refer to the sequel: The resolution of an atom with a definition must not instantiate the input arguments of the resolved atom. On the other hand, Gödel HL94 and ECLiPSe WWN97] use delay declarations, and SICStus Prolog [SIC97] employs block declarations (which are a special kind of delay declarations). Both delay and block declarations check the partial instantiation of some arguments of calls. For instance, the standard delay declaration for APPEND is

$$
d_{1}:=\text { delay app(Ls, _, _) until nonvar(Ls). }
$$

This declaration forbids the selection of an atom of the form app $(s, t, u)$ unless $s$ is a non-variable term, which is precisely what we need in order to run the queries $Q_{1}$ or $Q_{2}$ efficiently.

The adoption of dynamic scheduling has the disadvantage that various program properties that have been proven for logic and pure Prolog programs do not apply any longer.

The goal of our research is the study of termination properties. This is motivated by the fact that most of the literature on termination of logic programs (see De Schreye and Decorte [DD94] for a survey on this subject) assumes the 
standard Prolog selection rule, i.e., the leftmost one. Notable exceptions are Bezem Bez93 and Cavedon Cav89 who provide results for all selection rules. There are only few authors who tackled the specific problem of verifying the termination of logic programs with dynamic scheduling. Namely, Apt and Luitjes AL95, Marchiori and Teusink [MT95 and Smaus [Sma99b]. We compare our results with the ones in AL95, MT95, Sma99b] in the concluding section.

Another feature of logic programs which does not hold in presence of dynamic scheduling is the well-known switching lemma, which is, for instance, at the base of the result on the independence of the selection rule. In this paper we show that - under certain conditions - a weak form of the well-known switching lemma holds.

In order to recuperate at least part of the declarative reading of logic programming, we follow here the same approach to dynamic scheduling as Sma99b and we substitute the use of delay declarations by the restriction to inputconsuming derivations. The definition of input-consuming derivation is done in two phases. First we give the program a mode, that is, we partition the positions of each atom into input and output positions. Then, in presence of modes, input-consuming derivation steps are precisely those in which the input arguments of the selected atom will not be instantiated by the unification with the clause's head. If in a query no atom is resolvable via an input-consuming derivation step and a failure does not arise then we have a deadlock situation!.

For example, the standard mode for the program APPEND reported above, when used for concatenating two lists, is app(In, In, Out). Notice that in this case the delay declaration $d_{1}$ serves precisely the purpose of guaranteeing that if an atom of the form $\operatorname{app}(s, t, X)$ (with $X$ being a variable) is selectable and unifiable with a clause head, then the resulting derivation step is input-consuming.

It is also worth remarking that, as a large body of literature shows, the vast majority of "usual" programs are actually moded and are, in a well-defined sense consistent wrt. to their modes (e.g., well-moded, nicely-moded, simplymoded, etc.); see for example AP94b, AM94, or more simply, the tables of programs we report in Section 7, or consider for instance the logic programming language Mercury [SHC96], which requires that its programs are moded (and well-moded).

\section{Contributions of this paper}

In this paper we study some properties of input-consuming derivations.

In the first place we show that, if we restrict ourselves to programs and queries which are nicely-moded, then a weak form of the well-known switching lemma holds.

Furthermore, we study the termination properties of input-consuming derivations. For this we define the class of input terminating programs which characterizes programs whose input-consuming derivations starting in a nicely-moded

\footnotetext{
${ }^{1}$ As we discuss in Section 3.2, this notion of deadlock differs, in some way, from the usual one, which is given in the case of programs employing delay declarations.
} 
query are finite. In order to prove that a program is input terminating, we use the concept of quasi recurrent program (similar to, but noticeably less restrictive than the concept of semi-recurrent program introduced in AP94a). We show that if $P$ is nicely-moded and quasi recurrent then all its input-consuming derivations starting from a nicely-moded query terminate.

Furthermore, we demonstrate that under mild additional constraints (namely, simply-modedness and input-recurrency) the above condition is both sufficient and necessary for ensuring that all input-consuming derivations starting from a nicely-moded query terminate.

This approach generalizes the method described in Sma99b in two ways: First because we also provide conditions which are both necessary and sufficient, and secondly because we do not require programs and queries to be well-moded; we only assume that they are nicely-moded. This is actually crucial: When programs and queries are well-moded, derivations cannot deadlock. Thus, as opposed to Sma99b, our results capture also termination by deadlock. For instance, we can easily prove that the query $\operatorname{app}(X, Y, Z)$ terminates. A more detailed comparison is presented in the concluding section.

We also show that the results presented in this paper can be extended to programs and queries which are permutation nicely- or simply-moded, SHK98.

To evaluate the practicality of the results we present, we consider the programs from various well-known collections, and we check whether they satisfy the conditions of our main theorem.

The paper is organized as follows. Section 2 contains some preliminary notations and definitions. In Section 3 input-consuming derivations are introduced and some properties of them are proven. In Section 4 we prove that, for nicelymoded input-consuming programs, a left switching lemma holds. In Section 5 a method for proving input termination of programs is presented, first in a non-modular way, then for modular programs. In Section 6 we show that this method is necessary for the class of simply-moded and input-recursive programs. Section 1 discusses the applicability of our results through simple examples of programs and reports the results obtained by applying our method to various benchmarks. Finally, Section 8 concludes the paper.

\section{Preliminaries}

The reader is assumed to be familiar with the terminology and the basic results of logic programs Apt90, Apt97, Llo87.

\subsection{Terms and Substitutions}

Let $\mathcal{T}$ be the set of terms built on a finite set of data constructors $\mathcal{C}$ and a denumerable set of variable symbols $\mathcal{V}$. A substitution $\theta$ is a mapping from $\mathcal{V}$ to $\mathcal{T}$ such that $\operatorname{Dom}(\theta)=\{X \mid \theta(X) \neq X\}$ is finite. For any syntactic object $o$, we denote by $\operatorname{Var}(o)$ the set of variables occurring in $o$. A syntactic object is linear if every variable occurs in it at most once. We denote by $\epsilon$ the empty 
substitution. The composition $\theta \sigma$ of the substitutions $\theta$ and $\sigma$ is defined as the functional composition, i.e., $\theta \sigma(X)=\sigma(\theta(X))$. We consider the pre-ordering $\leq$ (more general than) on substitutions such that $\theta \leq \sigma$ iff there exists $\gamma$ such that $\theta \gamma=\sigma$. The result of the application of a substitution $\theta$ to a term $t$ is said an instance of $t$ and it is denoted by $t \theta$. We also consider the pre-ordering $\leq$ (more general than) on terms such that $t \leq t^{\prime}$ iff there exists $\theta$ such that $t \theta=t^{\prime}$. We denote by $\approx$ the associated equivalence relation (variance). A substitution $\theta$ is a unifier of terms $t$ and $t^{\prime}$ iff $t \theta=t^{\prime} \theta$. We denote by $m g u\left(t, t^{\prime}\right)$ any most general unifier (mgu, in short) of $t$ and $t^{\prime}$. An mgu $\theta$ of terms $t$ and $t^{\prime}$ is called relevant iff $\operatorname{Var}(\theta) \subseteq \operatorname{Var}(t) \cup \operatorname{Var}\left(t^{\prime}\right)$.

\subsection{Programs and Derivations}

Let $\mathcal{P}$ be a finite set of predicate symbols. An atom is an object of the form $p\left(t_{1}, \ldots, t_{n}\right)$ where $p \in \mathcal{P}$ is an $n$-ary predicate symbol and $t_{1}, \ldots, t_{n} \in \mathcal{T}$. Given an atom $A$, we denote by $\operatorname{Rel}(A)$ the predicate symbol of $A$. A query is a finite, possibly empty, sequence of atoms $A_{1}, \ldots, A_{m}$. The empty query is denoted by $\square$. Following the convention adopted in [Apt97, we use bold characters to denote queries. A clause is a formula $H \leftarrow \mathbf{B}$ where $H$ is an atom (the head) and $\mathbf{B}$ is a query (the body). When $\mathbf{B}$ is empty, $H \leftarrow \mathbf{B}$ is written $H \leftarrow$ and is called a unit clause. A program is a finite set of clauses. We denote atoms by $A, B, H, \ldots$, queries by $Q, \mathbf{A}, \mathbf{B}, \mathbf{C}, \ldots$, clauses by $c, d, \ldots$, and programs by $P$.

Computations are constructed as sequences of "basic" steps. Consider a non-empty query $\mathbf{A}, B, \mathbf{C}$ and a clause $c$. Let $H \leftarrow \mathbf{B}$ be a variant of $c$ variable disjoint from $\mathbf{A}, B, \mathbf{C}$. Let $B$ and $H$ unify with mgu $\theta$. The query $(\mathbf{A}, \mathbf{B}, \mathbf{C}) \theta$ is called a resolvent of $\mathbf{A}, B, \mathbf{C}$ and $c$ with selected atom $B$ and mgu $\theta$. A derivation step is denoted by

$$
\mathbf{A}, B, \mathbf{C} \stackrel{\theta}{\Longrightarrow} P, c(\mathbf{A}, \mathbf{B}, \mathbf{C}) \theta
$$

The clause $H \leftarrow \mathbf{B}$ is called its input clause. The atom $B$ is called the selected atom of $\mathbf{A}, B, \mathbf{C}$.

If $P$ is clear from the context or $c$ is irrelevant then we drop the reference to them. A derivation is obtained by iterating derivation steps. A maximal sequence

$$
\delta:=Q_{0} \stackrel{\theta_{1}}{\Longrightarrow}{ }_{P, c_{1}} Q_{1} \stackrel{\theta_{2}}{\Longrightarrow}{ }_{P, c_{2}} \cdots Q_{n}{\stackrel{\theta_{n+1}}{\Longrightarrow}}_{P, c_{n+1}} Q_{n+1} \cdots
$$

is called a derivation of $P \cup\left\{Q_{0}\right\}$ provided that for every step the standardization apart condition holds, i.e., the input clause employed is variable disjoint from the initial query $Q_{0}$ and from the substitutions and the input clauses used at earlier steps.

Derivations can be finite or infinite. If $\delta:=Q_{0}{\stackrel{\theta_{1}}{\Longrightarrow}}_{P, c_{1}} \cdots{\stackrel{\theta_{n}}{\Longrightarrow}}_{P, c_{n}} Q_{n}$ is a finite prefix of a derivation, also denoted $\delta:=Q_{0} \stackrel{\theta}{\longmapsto} Q_{n}$ with $\theta=\theta_{1} \cdots \theta_{n}$, we say that $\delta$ is a partial derivation and $\theta$ is a partial computed answer substitution of $P \cup\left\{Q_{0}\right\}$. If $\delta$ is maximal and ends with the empty query then $\theta$ is 
called computed answer substitution (c.a.s., for short). The length of a (partial) derivation $\delta$, denoted by len $(\delta)$, is the number of derivation steps in $\delta$.

The following definition of B-step is due to Smaus SSma99a.

Definition 1 (B-step) Let $\mathbf{A}, B, \mathbf{C} \stackrel{\theta}{\Longrightarrow}(\mathbf{A}, \mathbf{B}, \mathbf{C}) \theta$ be a derivation step. We say that each atom in $\mathbf{B} \theta$ is a direct descendant of $\mathrm{B}$, and for each atom $E$ in $(\mathbf{A}, \mathbf{C}), E \theta$ is a direct descendant of $\mathrm{E}$. We say that $E$ is a descendant of $F$ if the pair $(E, F)$ is in the reflexive, transitive closure of the relation is a direct descendant of. Consider a derivation $Q_{0} \stackrel{\theta_{1}}{\Longrightarrow} \cdots \stackrel{\theta_{i}}{\Longrightarrow} Q_{i} \cdots \stackrel{\theta_{j}}{\Longrightarrow} Q_{j} \stackrel{\theta_{j+1}}{\Longrightarrow}$ $Q_{j+1} \cdots$. We say that $Q_{j} \stackrel{\theta_{j+1}}{\Longrightarrow} Q_{j+1} \cdots$ is a $\mathbf{B}$-step if $\mathbf{B}$ is a subquery of $Q_{i}$ and the selected atom in $Q_{j}$ is a descendant of an atom in $\mathbf{B}$.

\section{Modes and Input-Consuming Derivations}

In this section we introduce the concept of input-consuming derivation which is strictly related to the notion of mode; we discuss the relations between inputconsuming derivations and programs using delay declarations; we recall the notion of nicely-moded program and state some properties.

\subsection{Input-Consuming Derivations}

Let us first recall the notion of mode. A mode is a function that labels as input or output the positions of each predicate in order to indicate how the arguments of a predicate should be used.

Definition 2 (Mode) Consider an n-ary predicate symbol $p$. A mode for $p$ is a function $m_{p}$ from $\{1, \ldots, n\}$ to $\{$ In, Out $\}$.

If $m_{p}(i)=$ In (resp. Out), we say that $i$ is an input (resp. output) position of $p$ (wrt. $m_{p}$ ). We assume that each predicate symbol has a unique mode associated to it; multiple modes may be obtained by simply renaming the predicates.

If $Q$ is a query, we denote by $\operatorname{In}(Q)($ resp. Out $(Q))$ the sequence of terms filling in the input (resp. output) positions of predicates in $Q$. Moreover, when writing an atom as $p(\mathbf{s}, \mathbf{t})$, we are indicating with $\mathbf{s}$ the sequence of terms filling in the input positions of $p$ and with $\mathbf{t}$ the sequence of terms filling in the output positions of $p$.

The notion of input-consuming derivation was introduced in Sma99b and is defined as follows.

\section{Definition 3 (Input-Consuming)}

- An atom $p(\mathbf{s}, \mathbf{t})$ is called input-consuming resolvable wrt. a clause $c:=$ $p(\mathbf{u}, \mathbf{v}) \leftarrow Q$ and a substitution $\theta$ iff $\theta=\operatorname{mgu}(p(\mathbf{s}, \mathbf{t}), p(\mathbf{u}, \mathbf{v}))$ and $\mathbf{s}=\mathbf{s} \theta$. 
- A derivation step

$$
\mathbf{A}, B, \mathbf{C} \stackrel{\theta}{\Longrightarrow}(\mathbf{A}, \mathbf{B}, \mathbf{C}) \theta
$$

is called input-consuming iff the selected atom $B$ is input-consuming resolvable wrt. the input clause $c$ and the substitution $\theta$.

- A derivation is called input-consuming iff all its derivation steps are inputconsuming.

The following lemma states that we are allowed to restrict our attention to input-consuming derivations with relevant mgu's.

Lemma 4 Let $p(\mathbf{s}, \mathbf{t})$ and $p(\mathbf{u}, \mathbf{v})$ be two atoms. If there exists an mgu $\theta$ of $p(\mathbf{s}, \mathbf{t})$ and $p(\mathbf{u}, \mathbf{v})$ such that $\mathbf{s} \theta=\mathbf{s}$, then there exists a relevant mgu $\vartheta$ of $p(\mathbf{s}, \mathbf{t})$ and $p(\mathbf{u}, \mathbf{v})$ such that $\mathbf{s} \vartheta=\mathbf{s}$.

Proof. Since $p(\mathbf{s}, \mathbf{t})$ and $p(\mathbf{u}, \mathbf{v})$ are unifiable, there exists a relevant mgu $\theta_{\text {rel }}$ of them (cfr. Apt97], Theorem 2.16). Now, $\theta_{\text {rel }}$ is a renaming of $\theta$. Thus $\mathbf{s} \theta_{\text {rel }}$ is a variant of $\mathbf{s}$. Then there exists a renaming $\rho$ such that $\operatorname{Dom}(\rho) \subseteq \operatorname{Var}(\mathbf{s}, \mathbf{t}, \mathbf{u}, \mathbf{v})$ and $\mathbf{s} \theta_{\text {rel }} \rho=\mathbf{s}$. Now, take $\vartheta=\theta_{\text {rel }} \rho$.

From now on, we assume that all mgu's used in the input-consuming derivation steps are relevant.

Example 5 Consider the program REVERSE with accumulator in the modes defined below.

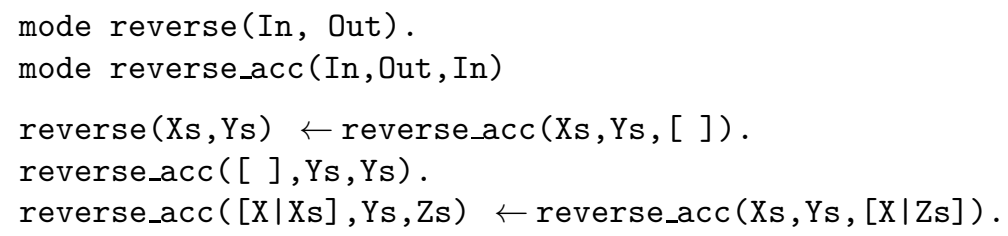

The derivation $\delta$ of $\mathrm{REVERSE} \cup\{$ reverse $([\mathrm{X} 1, \mathrm{X} 2], \mathrm{Zs})\}$ depicted below is inputconsuming.

$$
\begin{aligned}
\delta:=\quad & \text { reverse }([\mathrm{X} 1, \mathrm{X} 2], \mathrm{Zs}) \Rightarrow \text { reverse_acc }([\mathrm{X} 1, \mathrm{X} 2], \mathrm{Zs},[]) \Rightarrow \\
& \text { reverse_acc }([\mathrm{X} 2], \mathrm{Zs},[\mathrm{X} 1]) \Rightarrow \text { reverse_acc }([], \mathrm{Zs},[\mathrm{X} 2, \mathrm{X} 1]) \Rightarrow \square .
\end{aligned}
$$

\subsection{Input-Consuming vs. Delay Declarations}

Delay declarations are by far the most popular mechanism for implementing dynamic scheduling. However, being a non-logical mechanism, they are difficult to model and there are few proposals concerning their semantics [Mar97 and FGMP97.

An alternative approach to dynamic scheduling, which is much more declarative in nature, has been proposed by Smaus Sma99b. It consists in the use of input-consuming derivations. 
There is a main difference between the concept of delay declaration and the one of input-consuming derivation: While in the first case only the atom selectability is controlled, in the second one both the atom and the clause selectability are affected. In fact, in presence of delay declarations, if an atom is selectable then it can be resolved with respect to any program clause (provided it unifies with its head); on the contrary, in an input-consuming derivation, if an atom is selectable then it is input-consuming resolvable wrt. some, but not necessarily all, program clauses, i.e, only a restricted class of clauses can be used for resolution.

Also the concept of deadlock has to be understood in two different ways. For programs using delay declarations a deadlock situation occurs when no atom in a query satisfies the delay declarations (i.e., no atom is selectable), while for input-consuming derivations a deadlock occurs when no atom in a query is resolvable via an input-consuming derivation step and the derivation does not fail, i.e., there is some atom in the query which unifies with a clause head but the unification is not input-consuming.

In spite of these differences, in many situations there is a strict relation between programs using delay declarations and input-consuming derivations. This relation is studied by Smaus in his $\mathrm{PhD}$ thesis Sma99a. More precisely, Smaus proves a result that relates block declarations and input-consuming derivations. A block declaration is a special case of delay declaration and it is used to declare that certain arguments of an atom must be non-variable when the atom is selected for resolution. In Chapter 7 of [Sma99a], Smaus shows that block declarations can be used to ensure that derivations are input-consuming. In force of this result and of practical experience, we might claim that in most "usual" moded programs using them, delay declarations are employed precisely for ensuring the input-consumedness of the derivations.

In fact, delay declarations are generally employed to guarantee that the interpreter will not use an "inappropriate" clause for resolving an atom (the other, perhaps less prominent, use of delay declarations is to ensure absence of runtime errors, but we do not address this issue in this paper). This is achieved by preventing the selection of an atom until a certain degree of instantiation is reached. This degree of instantiation ensures then that the atom is unifiable only with the heads of the "appropriate" clauses. In presence of modes, we can reasonably assume that this degree of instantiation is the one of the input positions, which are the ones carrying the information. Now, it is easy to see that a derivation step involving a clause $c$ is input-consuming iff no further instantiation of the input positions of the resolved atom could prevent it from being resolvable with $c$. Therefore $c$ must belong to the set of "appropriate" clauses for resolving it. Thus, the concepts of input-consuming derivation and of delay declarations are often employed for ensuring the same properties.

\subsection{Nicely-Moded Programs}

In the sequel of the paper we will restrict ourselves to programs and queries which are nicely-moded. In this section we report the definition of this concept 
together with some basic important properties of nicely-moded programs.

\section{Definition 6 (Nicely-Moded)}

- A query $Q:=p_{1}\left(\mathbf{s}_{1}, \mathbf{t}_{1}\right), \ldots, p_{n}\left(\mathbf{s}_{n}, \mathbf{t}_{n}\right)$ is nicely-moded if $\mathbf{t}_{1}, \ldots, \mathbf{t}_{n}$ is a linear sequence of terms and for all $i \in\{1, \ldots, n\}$

$$
\operatorname{Var}\left(\mathbf{s}_{i}\right) \cap \bigcup_{j=i}^{n} \operatorname{Var}\left(\mathbf{t}_{j}\right)=\emptyset .
$$

- A clause $c=p\left(\mathbf{s}_{0}, \mathbf{t}_{0}\right) \leftarrow Q$ is nicely-moded if $Q$ is nicely-moded and

$$
\operatorname{Var}\left(\mathbf{s}_{0}\right) \cap \bigcup_{j=1}^{n} \operatorname{Var}\left(\mathbf{t}_{j}\right)=\emptyset .
$$

In particular, every unit clause is nicely-moded.

- A program $P$ is nicely-moded if all of its clauses are nicely-moded.

Note that a one-atom query $p(\mathbf{s}, \mathbf{t})$ is nicely-moded if and only if $\mathbf{t}$ is linear and $\operatorname{Var}(\mathbf{s}) \cap \operatorname{Var}(\mathbf{t})=\emptyset$.

\section{Example 7}

- The program APPEND in the modes app(In,In,Out) is nicely-moded.

- The program REVERSE with accumulator in the modes depicted in Example 5 is nicely-moded.

- The following program MERGE is nicely-moded.

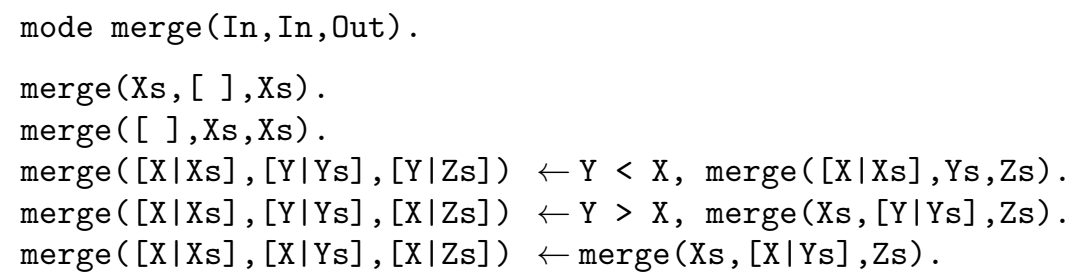

The following result is due to Smaus Sma99a, and states that the class of programs and queries we are considering is persistent under resolution.

Lemma 8 Every resolvent of a nicely-moded query $Q$ and a nicely-moded clause $c$, where the derivation step is input-consuming and $\operatorname{Var}(Q) \cap \operatorname{Var}(c)=\emptyset$, is nicely-moded.

The following Remark, also in Sma99a, is an immediate consequence of the definition of input-consuming derivation step and the fact that the mgu's we consider are relevant.

Remark 9 Let the program $P$ and the query $Q:=\mathbf{A}, p(\mathbf{s}, \mathbf{t}), \mathbf{C}$ be nicely-moded. If $\mathbf{A}, p(\mathbf{s}, \mathbf{t}), \mathbf{C} \stackrel{\theta}{\Longrightarrow}(\mathbf{A}, \mathbf{B}, \mathbf{C}) \theta$ is an input-consuming derivation step with selected atom $p(\mathbf{s}, \mathbf{t})$, then $\mathbf{A} \theta=\mathbf{A}$. 


\section{The Left Switching Lemma}

The switching lemma (see for instance [Apt97], Lemma 3.32) is a well-known result which allows one to prove the independence of the computed answer substitutions from the selection rule.

In the case of logic programs using dynamic scheduling, the switching lemma does not hold any longer. For example, in program APPEND reported in the introduction (together with the delay declaration $d_{1}$ ) we have that the rightmost atom of $Q_{2}$ is selectable only after the leftmost one has been resolved; i.e., the switching lemma cannot be applied.

Nevertheless we can show that, for input-consuming derivations of nicelymoded programs, a weak version of the switching lemma still holds. Intuitively, we show that we can switch the selection of two atoms whenever this results in a left to right selection. For this reason, we call it left switching lemma.

First, we need one technical result, stating that the only variables of a query that can be "affected" in an input-consuming derivation process are those occurring in some output positions. Intuitively, this means that if the input arguments of a call are not "sufficiently instantiated" then it is delayed until it allows for an input-consuming derivation step (if it is not the case then a deadlock situation will arise).

Lemma 10 Let the program $P$ and the query $Q$ be nicely-moded. Let $\delta:=$ $Q \stackrel{\theta}{\longmapsto} Q^{\prime}$ be a partial input-consuming derivation of $P \cup\{Q\}$. Then, for all $x \in \operatorname{Var}(Q)$ and $x \notin \operatorname{Var}(\operatorname{Out}(Q)), x \theta=x$.

Proof. Let us first establish the following claim.

Claim 11 Let $\mathbf{z}$ and $\mathbf{w}$ be two variable disjoint sequences of terms such that $\mathbf{w}$ is linear and $\theta=m g u(\mathbf{z}, \mathbf{w})$. If $s_{1}$ and $s_{2}$ are two variable disjoint terms occurring in $\mathbf{z}$ then $s_{1} \theta$ and $s_{2} \theta$ are variable disjoint terms.

Proof. The result follows from Lemmata 11.4 and 11.5 in AP94a.

We proceed with the proof of the lemma by induction on $\operatorname{len}(\delta)$.

Base Case. Let $\operatorname{len}(\delta)=0$. In this case $Q=Q^{\prime}$ and the result follows trivially.

Induction step. Let len $(\delta)>0$. Suppose that $Q:=\mathbf{A}, p(\mathbf{s}, \mathbf{t}), \mathbf{C}$ and

$$
\delta:=\mathbf{A}, p(\mathbf{s}, \mathbf{t}), \mathbf{C} \stackrel{\theta_{1}}{\Longrightarrow}(\mathbf{A}, \mathbf{B}, \mathbf{C}) \theta_{1} \stackrel{\theta_{2}}{\longmapsto} Q^{\prime}
$$

where $p(\mathbf{s}, \mathbf{t})$ is the selected atom of $Q, c:=p(\mathbf{u}, \mathbf{v}) \leftarrow \mathbf{B}$ is the input clause used in the first derivation step, $\theta_{1}$ is a relevant mgu of $p(\mathbf{s}, \mathbf{t})$ and $p(\mathbf{u}, \mathbf{v})$ and $\theta=\theta_{1} \theta_{2}$.

Let $x \in \operatorname{Var}(\mathbf{A}, p(\mathbf{s}, \mathbf{t}), \mathbf{C})$ and $x \notin \operatorname{Var}(\operatorname{Out}(\mathbf{A}, p(\mathbf{s}, \mathbf{t}), \mathbf{C}))$. We first show that

$$
x \theta_{1}=x
$$

We distinguish two cases. 
(a) $x \in \operatorname{Var}(\mathbf{s})$. In this case, property (1) follows from the hypothesis that $\delta$ is input-consuming.

(b) $x \notin \operatorname{Var}(\mathbf{s})$. Since $x \in \operatorname{Var}(\mathbf{A}, p(\mathbf{s}, \mathbf{t}), \mathbf{C})$, by standardization apart, we have that $x \notin \operatorname{Var}(p(\mathbf{u}, \mathbf{v}))$. Moreover, since $x \notin \operatorname{Var}(\operatorname{Out}(\mathbf{A}, p(\mathbf{s}, \mathbf{t}), \mathbf{C}))$, it also holds that $x \notin \operatorname{Var}(p(\mathbf{s}, \mathbf{t}))$. Then, property (11) follows from relevance of $\theta_{1}$.

Now we show that

$$
x \theta_{2}=x
$$

Again, we distinguish two cases:

(c) $x \notin \operatorname{Var}\left((\mathbf{A}, \mathbf{B}, \mathbf{C}) \theta_{1}\right)$. In this case, because of the standardization apart condition, $x$ will never occur in $(\mathbf{A}, \mathbf{B}, \mathbf{C}) \theta_{1} \stackrel{\theta_{2}}{\longmapsto} Q^{\prime}$. Hence, $x \notin \operatorname{Dom}\left(\theta_{2}\right)$ and $x \theta_{2}=x$.

(d) $x \in \operatorname{Var}\left((\mathbf{A}, \mathbf{B}, \mathbf{C}) \theta_{1}\right)$. In this case, in order to prove (2) we show that $x \notin \operatorname{Var}\left(\operatorname{Out}\left((\mathbf{A}, \mathbf{B}, \mathbf{C}) \theta_{1}\right)\right)$. The result then follows by the inductive hypothesis.

From the standardization apart, relevance of $\theta_{1}$ and the fact that the first derivation step is input-consuming, it follows that $\operatorname{Dom}\left(\theta_{1}\right) \cap \operatorname{Var}(Q) \subseteq \operatorname{Var}(\mathbf{t})$.

From the hypothesis that $Q$ is nicely-moded, $\operatorname{Var}(\mathbf{t}) \cap \operatorname{Var}(\operatorname{Out}(\mathbf{A}, \mathbf{C}))=\emptyset$. Hence, $\operatorname{Var}(\operatorname{Out}(\mathbf{A}, \mathbf{C})) \theta_{1}=\operatorname{Var}(\operatorname{Out}(\mathbf{A}, \mathbf{C}))$. Since $x \notin \operatorname{Var}(\operatorname{Out}(\mathbf{A}, \mathbf{C}))$, this proves that $x \notin \operatorname{Var}\left(\operatorname{Out}\left((\mathbf{A}, \mathbf{C}) \theta_{1}\right)\right)$.

It remains to be proven that $x \notin \operatorname{Var}\left(\operatorname{Out}\left(\mathbf{B} \theta_{1}\right)\right.$. We distinguish two cases.

$(d 1) x \notin \operatorname{Var}(\mathbf{s})$. Since $x \notin \operatorname{Var}(p(\mathbf{s}, \mathbf{t}))$, the fact that $x \notin \operatorname{Var}\left(\operatorname{Out}\left(\mathbf{B} \theta_{1}\right)\right.$ follows immediately by standardization apart condition and relevance of $\theta_{1}$.

$(d 2) x \in \operatorname{Var}(\mathbf{s})$. By known results (see [Apt97], Corollary 2.25), there exists two relevant mgu $\sigma_{1}$ and $\sigma_{2}$ such that

- $\theta_{1}=\sigma_{1} \sigma_{2}$,

- $\sigma_{1}=m g u(\mathbf{s}, \mathbf{u})$,

- $\sigma_{2}=m g u\left(\mathbf{t} \sigma_{1}, \mathbf{v} \sigma_{1}\right)$.

From relevance of $\sigma_{1}$ and the fact that, by nicely-modedness of $Q, \operatorname{Var}(\mathbf{s}) \cap$ $\operatorname{Var}(\mathbf{t})=\emptyset$, we have that $\mathbf{t} \sigma_{1}=\mathbf{t}$, and by the standardization apart condition $\operatorname{Var}(\mathbf{t}) \cap \operatorname{Var}\left(\mathbf{v} \sigma_{1}\right)=\emptyset$. Now by nicely-modedness of $c, \operatorname{Var}(\mathbf{u}) \cap \operatorname{Var}(\operatorname{Out}(\mathbf{B}))=$ $\emptyset$. Since $\sigma_{1}$ is relevant and by the standardization apart condition it follows that

$$
\operatorname{Var}\left(\mathbf{u} \sigma_{1}\right) \cap \operatorname{Var}\left(\operatorname{Out}\left(\mathbf{B} \sigma_{1}\right)\right)=\emptyset
$$

The proof proceeds now by contradiction. Suppose that $x \in \operatorname{Var}\left(\operatorname{Out}\left(\mathbf{B} \sigma_{1} \sigma_{2}\right)\right)$. Since by hypothesis $x \in \operatorname{Var}(\mathbf{s})$, and $\mathbf{s}=\mathbf{u} \sigma_{1} \sigma_{2}$, we have that $\operatorname{Var}\left(\mathbf{u} \sigma_{1} \sigma_{2}\right) \cap$ $\operatorname{Var}\left(\operatorname{Out}\left(\mathbf{B} \sigma_{1} \sigma_{2}\right)\right) \neq \emptyset$. By (3), this means that there exist two distinct variables $z_{1}$ and $z_{2}$ in $\operatorname{Var}\left(\sigma_{2}\right)$ such that $z_{1} \in \operatorname{Var}\left(\operatorname{Out}\left(\mathbf{B} \sigma_{1}\right)\right), z_{2} \in \operatorname{Var}\left(\mathbf{u} \sigma_{1}\right)$ and

$$
\operatorname{Var}\left(z_{1} \sigma_{2}\right) \cap \operatorname{Var}\left(z_{2} \sigma_{2}\right) \neq \emptyset
$$

Since, by the standardization apart condition and relevance of the mgu's, $\operatorname{Var}\left(\sigma_{2}\right)$ $\subseteq \operatorname{Var}\left(\mathbf{v} \sigma_{1}\right) \cup \operatorname{Var}(\mathbf{t})$ and $\left(\operatorname{Var}\left(\operatorname{Out}\left(\mathbf{B} \sigma_{1}\right)\right) \cup \operatorname{Var}\left(\mathbf{u} \sigma_{1}\right)\right) \cap \operatorname{Var}(\mathbf{t})=\emptyset$, we have 
that $z_{1}$ and $z_{2}$ are two disjoint subterms of $\mathbf{v} \sigma_{1}$. Since $\sigma_{2}=m g u\left(\mathbf{t}, \mathbf{v} \sigma_{1}\right), \mathbf{t}$ is linear and disjoint from $\mathbf{v} \sigma_{1}$, (4) contradicts Claim 11 .

The following corollary is an immediate consequence of the above lemma and the definition of nicely-moded program.

Corollary 12 Let the program $P$ and the one-atom query $A$ be nicely-moded. Let $\delta:=A \stackrel{\theta}{\longmapsto} Q^{\prime}$ be a partial input-consuming derivation of $P \cup\{A\}$. Then, for all $x \in \operatorname{Var}(\operatorname{In}(A)), x \theta=x$.

Next is the main result of this section, showing that for input-consuming nicely-moded programs one half of the well-known switching lemma holds.

Lemma 13 (Left-Switching) Let the program $P$ and the query $Q_{0}$ be nicelymoded. Let $\delta$ be a partial input-consuming derivation of $P \cup\left\{Q_{0}\right\}$ of the form

$$
\delta:=Q_{0} \stackrel{\theta_{1}}{\Longrightarrow} c_{1} Q_{1} \cdots Q_{n} \stackrel{\theta_{n+1}}{\Longrightarrow} c_{n+1} Q_{n+1}{\stackrel{\theta_{n+2}}{\Longrightarrow}}_{c_{n+2}} Q_{n+2}
$$

where

- $Q_{n}$ is a query of the form $\mathbf{A}, B, \mathbf{C}, D, \mathbf{E}$,

- $Q_{n+1}$ is a resolvent of $Q_{n}$ and $c_{n+1}$ wrt. $D$,

- $Q_{n+2}$ is a resolvent of $Q_{n+1}$ and $c_{n+2}$ wrt. $B \theta_{n+1}$.

Then, there exist $Q_{n+1}^{\prime}, \theta_{n+1}^{\prime}, \theta_{n+2}^{\prime}$ and a derivation $\delta^{\prime}$ such that

$$
\theta_{n+1} \theta_{n+2}=\theta_{n+1}^{\prime} \theta_{n+2}^{\prime}
$$

and

$$
\delta^{\prime}:=Q_{0} \stackrel{\theta_{1}}{\Longrightarrow} c_{1} Q_{1} \cdots Q_{n} \stackrel{\theta_{n+1}^{\prime}}{\Longrightarrow} c_{n+2} Q_{n+1}^{\prime}{\stackrel{\theta_{n+2}^{\prime}}{\Longrightarrow}}_{c_{n+1}} Q_{n+2}
$$

where $\delta^{\prime}$ is input-consuming and

- $\delta$ and $\delta^{\prime}$ coincide up to the resolvent $Q_{n}$,

- $Q_{n+1}^{\prime}$ is a resolvent of $Q_{n}$ and $c_{n+2}$ wrt. $B$,

- $Q_{n+2}$ is a resolvent of $Q_{n+1}^{\prime}$ and $c_{n+1}$ wrt. $D \theta_{n+1}^{\prime}$,

- $\delta$ and $\delta^{\prime}$ coincide after the resolvent $Q_{n+2}$.

Proof. Let $B:=p(\mathbf{s}, \mathbf{t}), D:=q(\mathbf{u}, \mathbf{v}), c_{n+1}:=q\left(\mathbf{u}^{\prime}, \mathbf{v}^{\prime}\right) \leftarrow \mathbf{D}$ and $c_{n+2}:=$ $p\left(\mathbf{s}^{\prime}, \mathbf{t}^{\prime}\right) \leftarrow \mathbf{B}$. Hence, $\theta_{n+1}=\operatorname{mgu}\left(q(\mathbf{u}, \mathbf{v}), q\left(\mathbf{u}^{\prime}, \mathbf{v}^{\prime}\right)\right)$ and

$$
\mathbf{u} \theta_{n+1}=\mathbf{u} \text {, since } \delta \text { is input-consuming. }
$$

By (5) and the fact that $Q_{n}$ is nicely-moded and $\theta_{n+1}$ is relevant, we have that $p(\mathbf{s}, \mathbf{t}) \theta_{n+1}=p(\mathbf{s}, \mathbf{t})$. Then, $\theta_{n+2}=\operatorname{mgu}\left(p(\mathbf{s}, \mathbf{t}) \theta_{n+1}, p\left(\mathbf{s}^{\prime}, \mathbf{t}^{\prime}\right)\right)=m g u\left(p(\mathbf{s}, \mathbf{t}), p\left(\mathbf{s}^{\prime}, \mathbf{t}^{\prime}\right)\right)$ and

$$
\mathbf{s} \theta_{n+2}=\mathbf{s} \text {, since } \delta \text { is input-consuming. }
$$


Moreover.2

$$
\theta_{n+1} \theta_{n+2}=\operatorname{mgu}\left\{p(\mathbf{s}, \mathbf{t})=p\left(\mathbf{s}^{\prime}, \mathbf{t}^{\prime}\right), q(\mathbf{u}, \mathbf{v})=q\left(\mathbf{u}^{\prime}, \mathbf{v}^{\prime}\right)\right\}=\theta_{n+2} \theta_{n+2}^{\prime}
$$

where

$$
\theta_{n+2}^{\prime}=m g u\left(q(\mathbf{u}, \mathbf{v}) \theta_{n+2}, q\left(\mathbf{u}^{\prime}, \mathbf{v}^{\prime}\right) \theta_{n+2}\right)=\operatorname{mgu}\left(q(\mathbf{u}, \mathbf{v}) \theta_{n+2}, q\left(\mathbf{u}^{\prime}, \mathbf{v}^{\prime}\right)\right) .
$$

We construct the derivation $\delta^{\prime}$ as follows.

$$
\delta^{\prime}:=Q_{0} \stackrel{\theta_{1}}{\Longrightarrow} c_{1} Q_{1} \cdots Q_{n} \stackrel{\theta_{n+1}^{\prime}}{\Longrightarrow} c_{n+2} Q_{n+1}^{\prime} \stackrel{\theta_{n+2}^{\prime}}{\Longrightarrow} c_{n+1} Q_{n+2}
$$

where

$$
\theta_{n+1}^{\prime}=\theta_{n+2}
$$

By (6), $Q_{n} \stackrel{\theta_{n+1}^{\prime}}{\Longrightarrow} c_{n+2} Q_{n+1}^{\prime}$ is an input-consuming derivation step. Observe now that

$$
\begin{aligned}
\mathbf{u} \theta_{n+1}^{\prime} \theta_{n+2}^{\prime} & =\mathbf{u} \theta_{n+2} \theta_{n+2}^{\prime}, & & \text { by (8) } \\
& =\mathbf{u} \theta_{n+1} \theta_{n+2}, & & \text { by } \\
& =\mathbf{u} \theta_{n+2}, & & \text { (by } \\
& =\mathbf{u} \theta_{n+1}^{\prime}, & & \text { (by }(8)) .
\end{aligned}
$$

This proves that $Q_{n+1}^{\prime} \stackrel{\theta_{n+2}^{\prime}}{\Longrightarrow} c_{n+1} Q_{n+2}^{\prime}$ is an input-consuming derivation step.

This result shows that it is always possible to proceed left-to-right to resolve the selected atoms. Notice that this is different than saying that the leftmost atom of a query is always resolvable: It can very well be the case that the leftmost atom is suspended and the one next to it is resolvable. However, if the leftmost atom of a query is not resolvable then we can state that the derivation will not succeed, i.e., either it ends by deadlock, or by failure or it is infinite.

It is important to notice that if we drop the nicely-modedness condition the above lemma would not hold any longer. For instance, it does not apply to the query $Q_{1}$ of the introduction which is not nicely-moded. In fact, the leftmost atom of $Q_{1}$ is resolvable only after the rightmost one has been resolved at least once.

The following immediate corollary will be used in the sequel.

Corollary 14 Let the program $P$ and the query $Q:=\mathbf{A}, \mathbf{B}$ be nicely-moded. Suppose that

$$
\delta:=\mathbf{A}, \mathbf{B} \stackrel{\theta}{\longmapsto} \mathbf{C}_{1}, \mathbf{C}_{2}
$$

that is a partial input-consuming derivation of $P \cup\{Q\}$ where $\mathbf{C}_{1}$ and $\mathbf{C}_{2}$ are obtained by partially resolving $\mathbf{A}$ and $\mathbf{B}$, respectively. Then there exists a partial input-consuming derivation

$$
\delta^{\prime}:=\mathbf{A}, \mathbf{B} \stackrel{\theta_{1}}{\longmapsto} \mathbf{C}_{1}, \mathbf{B} \theta_{1} \stackrel{\theta_{2}}{\longmapsto} \mathbf{C}_{1}, \mathbf{C}_{2}
$$

where all the $\mathbf{A}$-steps are performed in the prefix $\mathbf{A}, \mathbf{B} \stackrel{\theta_{1}}{\longmapsto} \mathbf{C}_{1}, \mathbf{B} \theta_{1}$ and $\theta=$ $\theta_{1} \theta_{2}$.

${ }^{2}$ We use the notation $m g u(E)$ to denote the mgu of a set of equations $E$, see Apt97. 


\section{Termination}

In this section we study the termination of input-consuming derivations. To this end we refine the ideas of Bezem Bez93 and Cavedon Cav89 who studied the termination of logic programs in a very strong sense, namely with respect to all selection rules, and of Smaus [Sma99b] who characterized terminating inputconsuming derivations of programs which are both well and nicely-moded.

\subsection{Input Terminating Programs}

We first introduce the key notion of this section.

Definition 15 (Input Termination) A program is called input terminating iff all its input-consuming derivations started in a nicely-moded query are finite.

The method we use in order to prove that a program is input terminating is based on the following concept of moded level mapping due to Etalle et al. EBC99.

Definition 16 (Moded Level Mapping) Let $P$ be a program and $\mathcal{B}_{P}^{\mathcal{E}}$ be the extended Herbrand base $\left.\right|^{3}$ for the language associated with $P$. A function || is a moded level mapping for $P$ iff:

- it is a function ||$: \mathcal{B}_{P}^{\mathcal{E}} \rightarrow \mathbf{N}$ from atoms to natural numbers;

- for any $\mathbf{t}$ and $\mathbf{u},|p(\mathbf{s}, \mathbf{t})|=|p(\mathbf{s}, \mathbf{u})|$.

For $A \in \mathcal{B}_{P}^{\mathcal{E}},|A|$ is the level of $A$.

The condition $|p(\mathbf{s}, \mathbf{t})|=|p(\mathbf{s}, \mathbf{u})|$ states that the level of an atom is independent from the terms in its output positions. There is actually a small yet important difference between this definition and the one in EBC99]: In [EBC99] the level mapping is defined on ground atoms only. Indeed, in [EBC99] only well-moded atoms are considered, i.e., atoms with ground terms in the input positions. Here, instead, we are considering nicely-moded atoms whose input positions can be filled in by (possibly) non-ground terms.

Example 17 Let us denote by TSize $(t)$ the term size of a term $t$, that is the number of function and constant symbols that occur in $t$.

- A moded level mapping for the program APPEND reported in the introduction is as follows:

$$
\left|\operatorname{app}\left(x_{s}, y_{s}, z_{s}\right)\right|=\operatorname{TSize}\left(x_{s}\right) .
$$

\footnotetext{
${ }^{3}$ The extended Herbrand base of $P$ is the set of equivalence classes of all (possibly nonground) atoms, modulo renaming, whose predicate symbol appears in $P$. As usual, an atom is identified with its equivalence class.
} 
- A moded level mapping for the program REVERSE with accumulator of Example 5 is the following:

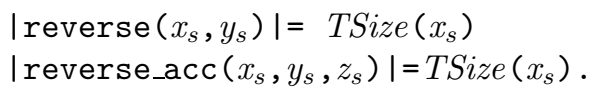

\subsection{Quasi Recurrency}

In order to give a sufficient condition for termination, we are going to employ a generalization of the concept of recurrent and of semi-recurrent program. The first notion (which in the case of normal programs, i.e., programs with negation, coincides with the one of acyclic program) was introduced in Bez93, AB91 and independently in [Cav91] in order to prove universal termination for all selection rules together with other properties of logic programs. Later, Apt and Pedreschi AP94a provided the new definition of semi-recurrent program, which is equivalent to the one of recurrent program, but it is easier to verify in an automatic fashion. In order to proceed, we need a preliminary definition.

Definition 18 Let $P$ be a program, $p$ and $q$ be relations. We say that $p$ refers to $q$ in $P$ iff there is a clause in $P$ with $p$ in the head and $q$ in the body. We say that $p$ depends on $q$ and write $p \sqsupseteq q$ in $P$ iff $(p, q)$ is in the reflexive and transitive closure of the relation refers to.

According to the above definition, $p \simeq q \equiv p \sqsubseteq q \wedge p \sqsupseteq q$ means that $p$ and $q$ are mutually recursive, and $p \sqsupset q \equiv p \sqsupseteq q \wedge p \neq q$ means that $p$ calls $q$ as a subprogram. Notice that $\sqsupset$ is a well-founded ordering.

Finally, we can provide the key concept we are going to use in order to prove input termination.

Definition 19 (Quasi Recurrency) Let $P$ be a program and ||$: \mathcal{B}_{P}^{\mathcal{E}} \rightarrow \mathbf{N}$ be a moded level mapping.

- A clause of $P$ is called quasi recurrent with respect to || if for every instance of it, $H \leftarrow \mathbf{A}, B, \mathbf{C}$

$$
\text { if } \operatorname{Rel}(H) \simeq \operatorname{Rel}(B) \text { then }|H|>|B| .
$$

- A program $P$ is called quasi recurrent with respect to || if all its clauses are. $P$ is called quasi recurrent if it is quasi recurrent wrt. some moded level mapping ||$: \mathcal{B}_{P}^{\mathcal{E}} \rightarrow \mathbf{N}$.

The notion of quasi recurrent program differs from the concepts of recurrent and of semi-recurrent program in two ways. First, we require that $|H|>|B|$ only for those body atoms which mutually depend on $\operatorname{Rel}(H)$; in contrast, both the concept of recurrent and of semi-recurrent program require that $|H|>|B|$ $(|H| \geq|B|$ in the case of semi-recurrency) also for the atoms for which $\operatorname{Rel}(H) \not$ $\operatorname{Rel}(B)$. Secondly, every instance of a program clause is considered, not only 
ground instances as in the case of (semi-)recurrent programs. This allows us to treat directly any nicely-moded query without introducing the concept of boundedness AP94a or cover as in MT95.

It is worthwhile noticing that this concept almost coincides with the one of ICD-acceptable program introduced and used in [Sma99b. We decided to use a different name because we believe that referring to the word acceptable might lead to confusion: The concept of acceptable program was introduced by Apt and Pedreschi [AP93, AP94a in order to prove termination of logic programs using the left-to-right selection rule. The crucial difference between recurrency and acceptability lies in the fact that the latter relies on a model $M$; this allows condition (9) to be checked only for those body atoms which are in a way "reachable" wrt. $M$. Hence, every recurrent program is acceptable but not vice-versa. As an aside, Marchiori and Teusink [MT95 introduce the notion of delay recurrent program although their concept is based on the presence of a model $M$. Our definition does not rely on a model, and so it is much more related to the notion of recurrent than the one of acceptable program.

We can now state our first basic result on termination, in the case of nonmodular programs.

Theorem 20 Let $P$ be a nicely-moded program. If $P$ is quasi recurrent then $P$ is input terminating.

Proof. It will be obtained from the proof of Theorem 24 by setting $R=\emptyset$.

Example 21 Consider the program MERGE defined in Example 7 . Let || be the moded level mapping for MERGE defined by

$$
\left|\operatorname{merge}\left(x_{s}, y_{s}, z_{s}\right)\right|=\operatorname{TSize}\left(x_{s}\right)+\operatorname{TSize}\left(y_{s}\right) \text {. }
$$

It is easy to prove that MERGE is quasi recurrent wrt. the moded level mapping above. By Theorem 20, all input-consuming derivations of MERGE started with a query merge $(s, t, u)$, where $u$ is linear and variable disjoint from $s$ and $t$, are terminating.

\subsection{Modular Termination}

This section contains a generalization of Theorem 20 to the modular case, as well as the complete proofs for it. The following lemma is a crucial one.

Lemma 22 Let the program $P$ and the query $Q:=A_{1}, \ldots, A_{n}$ be nicely-moded. Suppose that there exists an infinite input-consuming derivation $\delta$ of $P \cup\{Q\}$. Then, there exist an index $i \in\{1, \ldots, n\}$ and substitution $\theta$ such that

1. there exists an input-consuming derivation $\delta^{\prime}$ of $P \cup\{Q\}$ of the form

$$
\delta^{\prime}:=A_{1}, \ldots, A_{n} \stackrel{\theta}{\longmapsto} \mathbf{C},\left(A_{i}, \ldots, A_{n}\right) \theta \longmapsto \cdots
$$

2. there exists an infinite input-consuming derivation of $P \cup\left\{A_{i} \theta\right\}$. 
Proof. Let $\delta:=A_{1}, \ldots, A_{n} \longmapsto \cdots$ be an infinite input-consuming derivation of $P \cup\{Q\}$. Then $\delta$ contains an infinite number of $A_{k}$-steps for some $k \in$ $\{1, \ldots, n\}$. Let $i$ be the minimum of such $k$. Hence $\delta$ contains a finite number of $A_{j}$-steps for $j \in\{1, \ldots, i-1\}$ and there exists $\mathbf{C}$ and $\mathbf{D}$ such that

$$
\delta:=A_{1}, \ldots, A_{n} \stackrel{\vartheta}{\longmapsto} \mathbf{C}, \mathbf{D} \longmapsto \cdots
$$

where $A_{1}, \ldots, A_{n} \stackrel{\vartheta}{\longmapsto} \mathbf{C}, \mathbf{D}$ is a finite prefix of $\delta$ which comprises all the $A_{j^{-}}$ steps of $\delta$ for $j \in\{1, \ldots, i-1\}$ and $\mathbf{C}$ is the subquery of $\mathbf{C}, \mathbf{D}$ consisting of the atoms resulting from some $A_{j}$-step $(j \in\{1, \ldots, i-1\})$. By Corollary 14, there exists an infinite input-consuming derivation $\delta^{\prime}$ such that

$$
\delta^{\prime}:=A_{1}, \ldots, A_{n} \stackrel{\theta}{\longmapsto} \mathbf{C},\left(A_{i}, \ldots, A_{n}\right) \theta \stackrel{\theta^{\prime}}{\longmapsto} \mathbf{C}, \mathbf{D} \longmapsto \cdots
$$

where $\vartheta=\theta \theta^{\prime}$. This proves (i).

Now, let $\delta^{\prime \prime}:=\mathbf{C},\left(A_{i}, \ldots, A_{n}\right) \theta \stackrel{\theta^{\prime}}{\longmapsto} \mathbf{C}, \mathbf{D} \longmapsto \cdots$. Note that in $\delta^{\prime \prime}$ the atoms of $\mathbf{C}$ will never be selected and, by Remark 9, will never be instantiated. Let $\delta^{\prime \prime \prime}$ be obtained from $\delta^{\prime \prime}$ by omitting the prefix $\mathbf{C}$ in each query. Hence $\delta^{\prime \prime \prime}$ is an infinite input-consuming derivation of $P \cup\left\{\left(A_{i}, \ldots, A_{n}\right) \theta\right\}$ where an infinite number of $A_{i} \theta$-steps are performed. Again, By Remark 9 , for every finite prefix of $\delta^{\prime \prime \prime}$ of the form

$$
A_{i} \theta,\left(A_{i+1}, \ldots, A_{n}\right) \theta \stackrel{\sigma_{1}}{\longmapsto} \mathbf{D}_{1}, \mathbf{D}_{2} \stackrel{\sigma_{2}}{\Longrightarrow} \mathbf{D}_{1}^{\prime}, \mathbf{D}_{2}^{\prime}
$$

where $\mathbf{D}_{1}$ and $\mathbf{D}_{2}$ are obtained by partially resolving $A_{i} \theta$ and $\left(A_{i+1}, \ldots, A_{n}\right) \theta$, respectively, and $\mathbf{D}_{1}, \mathbf{D}_{2} \stackrel{\sigma_{2}}{\Longrightarrow} \mathbf{D}_{1}^{\prime}, \mathbf{D}_{2}^{\prime}$ is an $A_{j}$-step for some $j \in\{i+1, \ldots, n\}$, we have that $\mathbf{D}_{1}^{\prime}=\mathbf{D}_{1}$. Hence, from the hypothesis that there is an infinite number of $A_{i} \theta$-steps in $\delta^{\prime \prime}$, it follows that there exists an infinite input-consuming derivation of $P \cup\left\{A_{i} \theta\right\}$. This proves (ii).

The importance of the above lemma is shown by the following corollary of it, which will allow us to concentrate on queries containing only one atom.

Corollary 23 Let $P$ be a nicely-moded program. $P$ is input terminating iff for each nicely-moded one-atom query A all input-consuming derivations of $P \cup\{A\}$ are finite.

We can now state the main result of this section. Here and in what follows we say that a relation $p$ is defined in the program $P$ if $p$ occurs in a head of a clause of $P$, and that $P$ extends the program $R$ if no relation defined in $P$ occurs in $R$.

Theorem 24 Let $P$ and $R$ be two programs such that $P$ extends $R$. Suppose that

- $R$ is input terminating,

- $P$ is nicely-moded and quasi recurrent wrt. a moded level mapping || : $\mathcal{B}_{P}^{\mathcal{E}} \rightarrow \mathbf{N}$. 
Then $P \cup R$ is input terminating.

Proof. First, for each predicate symbol $p$, we define $\operatorname{dep}_{P}(p)$ to be the number of predicate symbols it depends on. More formally, $\operatorname{dep}_{P}(p)$ is defined as the cardinality of the set $\{q \mid q$ is defined in $P$ and $p \sqsupseteq q\}$. Clearly, $\operatorname{dep}_{P}(p)$ is always finite. Further, it is immediate to see that if $p \simeq q$ then $\operatorname{dep}_{P}(p)=$ $\operatorname{dep}_{P}(q)$ and that if $p \sqsupset q$ then $\operatorname{dep}_{P}(p)>\operatorname{dep}_{P}(q)$.

We can now prove our theorem. By Corollary 23, it is sufficient to prove that for any nicely-moded one-atom query $A$, all input-consuming derivations of $P \cup\{A\}$ are finite.

First notice that if $A$ is defined in $R$ then the result follows immediately from the hypothesis that $R$ is input terminating and that $P$ is an extension of $R$. So we can assume that $A$ is defined in $P$.

For the purpose of deriving a contradiction, assume that $\delta$ is an infinite input-consuming derivation of $(P \cup R) \cup\{A\}$ such that $A$ is defined in $P$. Then

$$
\delta:=A \stackrel{\theta_{1}}{\Longrightarrow}\left(B_{1}, \ldots, B_{n}\right) \theta_{1} \stackrel{\theta_{2}}{\Longrightarrow} \cdots
$$

where $H \leftarrow B_{1}, \ldots, B_{n}$ is the input clause used in the first derivation step and $\theta_{1}=m g u(A, H)$. Clearly, $\left(B_{1}, \ldots, B_{n}\right) \theta_{1}$ has an infinite input-consuming derivation in $P \cup R$. By Lemma 22, for some $i \in\{1, \ldots, n\}$ and for some substitution $\theta_{2}$,

1. there exists an infinite input-consuming derivation of $(P \cup R) \cup\{A\}$ of the form

$$
A \stackrel{\theta_{1}}{\Longrightarrow}\left(B_{1}, \ldots, B_{n}\right) \theta_{1} \stackrel{\theta_{2}}{\longrightarrow} \mathbf{C},\left(B_{i}, \ldots, B_{n}\right) \theta_{1} \theta_{2} \cdots ;
$$

2. there exists an infinite input-consuming derivation of $P \cup\left\{B_{i} \theta_{1} \theta_{2}\right\}$.

Notice also that $B_{i} \theta_{1} \theta_{2}$ is nicely-moded. Let now $\theta=\theta_{1} \theta_{2}$. Note that $H \theta \leftarrow$ $\left(B_{1}, \ldots, B_{n}\right) \theta$ is an instance of a clause of $P$.

We show that $(2)$ cannot hold. This is done by induction on $\left\langle\operatorname{dep}_{P}(\operatorname{Rel}(A)),|A|\right\rangle$ wrt. the ordering $\succ$ defined by: $\langle m, n\rangle \succ\left\langle m^{\prime}, n^{\prime}\right\rangle$ iff either $m>m^{\prime}$ or $m=m^{\prime}$ and $n>n^{\prime}$.

Base. Let $\operatorname{dep}_{P}(\operatorname{Rel}(A))=0(|A|$ is arbitrary). In this case, $A$ does not depend on any predicate symbol of $P$, thus all the $B_{i}$ as well as all the atoms occurring in its descendents in any input-consuming derivation are defined in $R$. The hypothesis that $R$ is input terminating contradicts (2) above.

Induction step. We distinguish two cases:

1. $\operatorname{Rel}(H) \sqsupset \operatorname{Rel}\left(B_{i}\right)$,

2. $\operatorname{Rel}(H) \simeq \operatorname{Rel}\left(B_{i}\right)$.

In case $(a)$ we have that $\operatorname{dep}_{P}(\operatorname{Rel}(A))=\operatorname{dep}_{P}(\operatorname{Rel}(H \theta))>\operatorname{dep}_{P}\left(\operatorname{Rel}\left(B_{i} \theta\right)\right)$. So, $\left\langle\operatorname{dep}_{P}(\operatorname{Rel}(A)),|A|\right\rangle=\left\langle\operatorname{dep}_{P}(\operatorname{Rel}(H \theta)),|H \theta|\right\rangle \succ\left\langle\operatorname{dep}_{P}\left(\operatorname{Rel}\left(B_{i} \theta\right)\right),\left|B_{i} \theta\right|\right\rangle$. In case $(b)$, from the hypothesis that $P$ is quasi recurrent wrt. || , it follows that $|H \theta|>\left|B_{i} \theta\right|$. 
Consider now the partial input-consuming derivation $A \stackrel{\theta}{\longmapsto} \mathbf{C},\left(B_{i}, \ldots, B_{n}\right) \theta$. By Corollary 12 and the fact that || is a moded level mapping, it follows that $|A|=|A \theta|=|H \theta|$. Therefore, $\left\langle\operatorname{dep}_{P}(\operatorname{Rel}(A)),|A|\right\rangle=\left\langle\operatorname{dep}_{P}(\operatorname{Rel}(H \theta)),|H \theta|\right\rangle \succ$ $\left\langle\operatorname{dep}_{P}\left(\operatorname{Rel}\left(B_{i} \theta\right)\right),\left|B_{i} \theta\right|\right\rangle$. In both cases, the contradiction follows by the inductive hypothesis.

Example 25 The program FLATTEN using difference-lists is nicely-moded with respect to the modes described below, provided that one replaces "I" by ",", as we have done here.

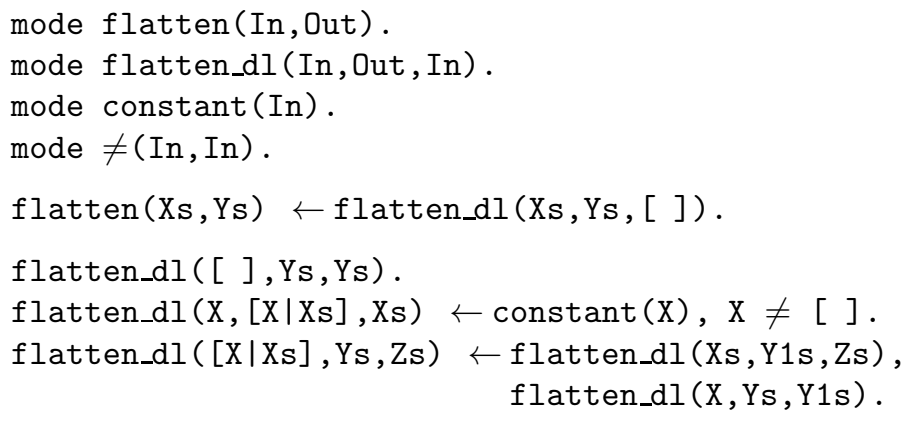

Consider the moded level mapping for FLATTEN defined by

$$
\begin{aligned}
& \left|f \operatorname{flatten}\left(x_{s}, y_{s}\right)\right|=\operatorname{TSize}\left(x_{s}\right) \\
& \mid \text { flatten_dl }\left(x_{s}, y_{s}, z_{s}\right) \mid=\operatorname{TSize}\left(x_{s}\right) .
\end{aligned}
$$

It is easy to see that the program FLATTEN is quasi recurrent wrt. the moded level mapping above. Hence, all input-consuming derivations of program FLATTEN started with a query flatten $(s, t)$, where $t$ is linear and variable disjoint from $s$, are terminating.

\section{Termination: A Necessary Condition}

Theorem 20 provides a sufficient condition for termination. The condition is not necessary, as demonstrated by the following simple example.

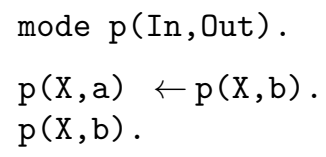

This program is clearly input terminating, however it is not quasi recurrent. If it was, we would have that $|\mathrm{p}(\mathrm{X}, \mathrm{a})|>|\mathrm{p}(\mathrm{X}, \mathrm{b})|$, for some moded level mapping I | (otherwise the first clause would not be quasi recurrent). On the other hand, since $\mathrm{p}(\mathrm{X}, \mathrm{a})$ and $\mathrm{p}(\mathrm{X}, \mathrm{b})$ differ only for the terms filling in their output positions, by definition of moded level mapping, $|\mathrm{p}(\mathrm{X}, \mathrm{a})|=|\mathrm{p}(\mathrm{X}, \mathrm{b})|$. Hence, we have a contradiction. 
Nevertheless, as shown by other works, e.g., Bez93, AP93, EBC99], it is important to be able to give a characterization of termination, i.e., a condition which is necessary and sufficient to ensure termination. To this purpose is dedicated this section.

\subsection{Simply-Moded Programs}

As demonstrated by the example above, in order to provide a necessary condition for termination we need to further restrict the class of programs we consider. The first problem is that we should rule out those situations in which termination is guaranteed by the instantiation of the output positions of some selected atom, as it happens in the above example. For this we restrict to simply-moded programs which are nicely-moded programs with the additional condition that the output arguments of clause bodies are variables.

\section{Definition 26 (Simply-Moded)}

- A query $Q$ (resp., a clause $c=H \leftarrow Q$ ) is simply-moded if it is nicelymoded and $\operatorname{Out}(Q)$ is a linear sequence of variables.

- A program $P$ is simply-moded iff all of its clauses are simply-moded.

It is important to notice that most programs are simply-moded (see the mini-survey at the end of AP93]) and that often non simply-moded programs can naturally be transformed into simply-moded ones.

\section{Example 27}

- The programs REVERSE of Example 月, MERGE of Example 1 and FLATTEN of Example 25 are all simply-moded.

- Consider the program LAST which extends REVERSE:

mode last(In, Out).

$\operatorname{last}(\mathrm{Ls}, \mathrm{E}) \leftarrow \operatorname{reverse}(\mathrm{Ls},[\mathrm{E} \mid]])$.

This program is not simply-moded since the argument filling in the output position in the body of the first clause is not a variable. However, it can be transformed into a simply-moded one as follows:

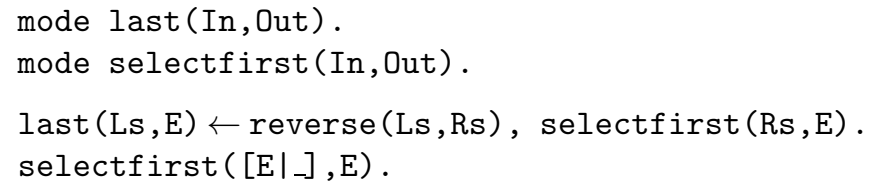

The following lemma, which is an immediate consequence of Lemma 30 in [AL95], shows the persistence of the notion of simply-modedness.

Lemma 28 Every resolvent of a simply-moded query $Q$ and a simply-moded clause $c$, where the derivation step is input-consuming and $\operatorname{Var}(Q) \cap \operatorname{Var}(c)=\emptyset$, is simply-moded. 


\subsection{Input-Recursive Programs}

Unfortunately, the restriction to simply-moded programs alone is not sufficient to extend Theorem 20 by a necessary condition. Consider for instance the following program QUICKSORT:

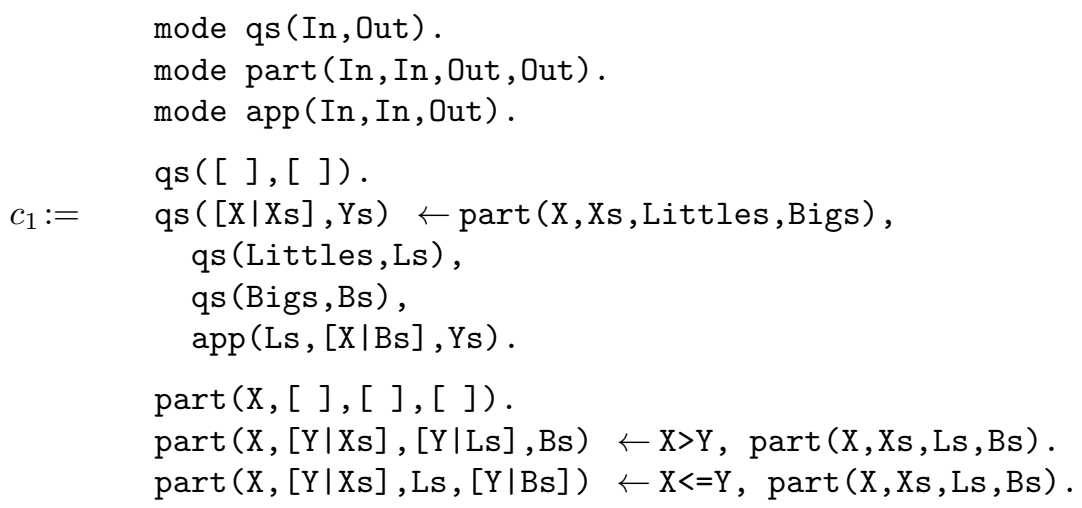

This program is simply-moded and input terminating quasi recurrent. Indeed, there exist no moded level mapping || such that, for every variable-instance, $|\mathrm{qs}([\mathrm{X} \mid \mathrm{Xs}], \mathrm{Ys})|>\mid \mathrm{qs}($ Littles, Ls $) \mid$ and $|\mathrm{qs}([\mathrm{X} \mid \mathrm{Xs}], \mathrm{Ys})|>$ $|q s(B i g s, B s)|$. This is due to the fact that, in clause $c_{1}$ there is no direct link between the input arguments of the recursive calls and those of the clause head. This motivates the following definition of input-recursive programs.

Definition 29 (Input-Recursive) Let $P$ be a program.

- A clause $H \leftarrow \mathbf{A}, B, \mathbf{C}$ of $P$ is called input-recursive if

$$
\text { if } \operatorname{Rel}(H) \simeq \operatorname{Rel}(B) \text { then } \operatorname{Var}(\operatorname{In}(B)) \subseteq \operatorname{Var}(\operatorname{In}(H)) \text {. }
$$

- A program $P$ is called input-recursive if all its clauses are.

Thus, we say that a clause is input-recursive if the set of variables occurring in the arguments filling in the input positions of each recursive call in the clause body is a subset of the set of variables occurring in the arguments filling in the input positions of the clause head. Input-recursive programs have strong similarities with primitive recursive functions.

\section{Example 30}

- The programs APPEND of the introduction, REVERSE of Example 5 and MERGE of Example 7 are all input-recursive.

\footnotetext{
${ }^{4}$ Provided that one models the built-in predicates $>$ and $<=$ as being defined by (an infinite number of) ground facts of the form $>(m, n)$ and $<=(m, n)$. The problem here is that the definition of input-consuming derivation does not consider the presence of built-ins.
} 
- The program FLATTEN of Example 25 is not input-recursive. This is due to the presence of the fresh variable Y1s in a body atom of the last clause.

- QUICKSORT, is not input-recursive. In particular, clause $c_{1}$ is not inputrecursive.

\subsection{Characterizing Input Terminating Programs}

We can now prove that by restricting ourselves to input-recursive and simplymoded programs, the condition of Theorem 20 is also a necessary one.

To prove this, we follow the approach of Apt and Pedreschi when characterizing terminating programs AP94a. First we introduce the notion of $I C$-tree that corresponds to the notion of $S$-tree in AP94a and provides us with a representation for all input-consuming derivations of a program $P$ with a query $Q$, then we define a level mapping which associates to every atom $A$ the number of nodes of a given IC-tree and finally we prove that $P$ is quasi recurrent wrt. such a level mapping.

Definition 31 (IC-tree) An IC-tree for $P \cup\{Q\}$ is a tree whose nodes are labelled with queries such that

- its branches are input-consuming derivations of $P \cup\{Q\}$,

- every node $Q$ has exactly one descendant for every atom $A$ of $Q$ and every clause $c$ from $P$ such that $A$ is input-consuming resolvable wrt. $c$. This descendant is a resolvent of $Q$ and $c$ wrt. A.

In this tree, a node's children consist of all its resolvents, "modulo renaming", via an input-consuming derivation step wrt. all the possible choices of a program clause and a selected atom.

Lemma 32 (IC-tree 1) An IC-tree for $P \cup\{Q\}$ is finite iff all input-consuming derivations of $P \cup\{Q\}$ are finite.

Proof. By definition, the IC-trees are finitely branching. The claim now follows by König's Lemma.

Notice that if an IC-tree for $P \cup\{Q\}$ is finite then all the IC-trees for $P \cup\{Q\}$ are finite.

For a program $P$ and a query $Q$, we denote by $\operatorname{nodes}_{P}^{i c}(Q)$ the number of nodes in an IC-tree for $P \cup\{Q\}$. The following properties of IC-trees will be needed.

Lemma 33 (IC-tree 2) Let $P$ be a program, $Q$ be a query and $T$ be a finite IC-tree for $P \cup\{Q\}$. Then

(i) for all non-root nodes $Q^{\prime}$ in $T$, $\operatorname{nodes}_{P}^{i c}\left(Q^{\prime}\right)<\operatorname{nodes}_{P}^{i c}(Q)$,

(ii) for all atoms $A$ of $Q$, nodes ${ }_{P}^{i c}(A) \leq \operatorname{nodes}_{P}^{i c}(Q)$. 
Proof. Immediate by Definition 31 of IC-tree.

We can now prove the desired result.

Theorem 34 Let $P$ be a simply-moded and input-recursive program. If $P$ is input terminating then $P$ is quasi recurrent.

Proof. We show that there exists a moded level mapping || for $P$ such that $P$ is quasi recurrent wrt. ||.

Given an atom $A$, we denote with $A^{*}$ an atom obtained from $A$ by replacing the terms filling in its output positions with fresh distinct variables. Clearly, we have that $A^{*}$ is simply-moded. Then we define the following moded level mapping for $P$ :

$$
|A|=\operatorname{nodes}_{P}^{i c}\left(A^{*}\right) .
$$

Notice that, the level $|A|$ of an atom $A$ is independent from the terms filling in its output positions, i.e., || is a moded level mapping. Moreover, since $P$ is input terminating and $A^{*}$ is simply-moded (in particular, it is nicely-moded), all the input-consuming derivations of $P \cup\left\{A^{*}\right\}$ are finite. Therefore, by Lemma 32, $\operatorname{nodes}_{P}^{i c}\left(A^{*}\right)$ is defined (and finite), and thus $|A|$ is defined (and finite) for every atom $A$.

We now prove that $P$ is quasi recurrent wrt. $\mid 1$.

Let $c: H \leftarrow \mathbf{A}, B, \mathbf{C}$ be a clause of $P$ and $H \theta \leftarrow \mathbf{A} \theta, B \theta, \mathbf{C} \theta$ be an instance of $c$ (for some substitution $\theta$ ). We show that if $\operatorname{Rel}(H) \simeq \operatorname{Rel}(B)$ then $|H \theta|>|B \theta|$.

Let $H=p(\mathbf{s}, \mathbf{t})$. Hence, $(H \theta)^{*}=p(\mathbf{s} \theta, \mathbf{x})$ where $\mathbf{x}$ is a sequence of fresh distinct variables. Consider a variant $c^{\prime}: H^{\prime} \leftarrow \mathbf{A}^{\prime}, B^{\prime}, \mathbf{C}^{\prime}$ of $c$ variable disjoint from $(H \theta)^{*}$. Let $\rho$ be a renaming such that $c^{\prime}=c \rho$. Clearly, $(H \theta)^{*}$ and $H^{\prime}$ unify. Let $\mu=m g u\left((H \theta)^{*}, H^{\prime}\right)=m g u\left((H \theta)^{*}, H \rho\right)=m g u(p(\mathbf{s} \theta, \mathbf{x}), p(\mathbf{s}, \mathbf{t}) \rho)$. By properties of substitutions (see Apt97]), since $\mathbf{x}$ consists of fresh variables, there exists two relevant mgu $\sigma_{1}$ and $\sigma_{2}$ such that

- $\sigma_{1}=m g u(\mathbf{s} \theta, \mathbf{s} \rho)$,

- $\sigma_{2}=m g u\left(\mathbf{x} \sigma_{1}, \mathbf{t} \rho \sigma_{1}\right)$.

Since $\mathbf{s} \rho \leq \mathbf{s} \theta$, we can assume that $\operatorname{Dom}\left(\sigma_{1}\right) \subseteq \operatorname{Var}(\mathbf{s} \rho)$. Because of standardization apart, since $\mathbf{x}$ consists of fresh variables, $\mathbf{x} \sigma_{1}=\mathbf{x}$ and thus $\sigma_{2}=$ $m g u\left(\mathbf{x}, \mathbf{t} \rho \sigma_{1}\right)$. Since $\mathbf{x}$ is a sequence of variables, we can also assume that $\operatorname{Dom}\left(\sigma_{2}\right) \subseteq \operatorname{Var}(\mathbf{x})$. Therefore $\operatorname{Dom}(\mu) \subseteq \operatorname{Var}\left(\operatorname{Out}\left((H \theta)^{*}\right)\right) \cup \operatorname{Var}(\operatorname{In}(H \rho))$. Moreover, since $\left(\mathbf{A}^{\prime}, B^{\prime}, \mathbf{C}^{\prime}\right) \mu=(\mathbf{A}, B, \mathbf{C}) \rho \mu$, we have that

$$
(H \theta)^{*} \stackrel{\mu}{\Longrightarrow}(\mathbf{A}, B, \mathbf{C}) \rho \mu
$$

is an input-consuming derivation step, i.e., $(\mathbf{A}, B, \mathbf{C}) \rho \mu$ is a descendant of $(H \theta)^{*}$ in an IC-tree for $P \cup\left\{(H \theta)^{*}\right\}$.

By definition of $\mu, \mathbf{s} \theta=\mathbf{s} \rho \mu$; hence

$$
(\rho \mu)_{\mid \operatorname{In}(H)}=\theta_{\mid \mathbf{s}} .
$$


Let now $B=p(\mathbf{u}, \mathbf{v})$. By (10) and the hypothesis that $c$ is input-recursive, that is $\operatorname{Var}(\operatorname{In}(B)) \subseteq \operatorname{Var}(\operatorname{In}(H))=\operatorname{Var}(\mathbf{s})$, it follows that

$$
\mathbf{u} \rho \mu=\mathbf{u}(\rho \mu)_{\mid \operatorname{In}(H)}=\mathbf{u} \theta_{\mid \mathbf{s}}=\mathbf{u} \theta .
$$

Moreover, since $c^{\prime}$ is simply-moded, $\operatorname{In}(H \rho) \cap \operatorname{Out}(B \rho)=\emptyset$. Hence, by definition of $\mu$ and standardization apart, $\operatorname{Dom}(\mu) \cap \operatorname{Out}(B \rho)=\emptyset$, i.e.,

$$
\mathbf{v} \rho \mu=\mathbf{v} \rho \text {. }
$$

Therefore, by (11) and (12), $B \rho \mu=p(\mathbf{u}, \mathbf{v}) \rho \mu=p(\mathbf{u} \theta, \mathbf{v} \rho)=(B \theta)^{*}$, i.e.,

$$
B \rho \mu=(B \theta)^{*} .
$$

Hence,

$$
\begin{aligned}
|H \theta| & =\operatorname{nodes}_{P}^{i c}\left((H \theta)^{*}\right) & & \text { by definition of }|| \\
& >\operatorname{nodes}_{P}^{i c}((\mathbf{A}, B, \mathbf{C}) \rho \mu) & & \text { by Lemma } 33(\mathrm{i}) \\
& \geq \operatorname{nodes}_{P}^{i c}(B \rho \mu) & & \text { by Lemma } 33(\mathrm{ii}) \\
& =\operatorname{nodes}_{P}^{i c}\left((B \theta)^{*}\right) & & \text { by (13) } \\
& =|B \theta| & & \text { by definition of }|| .
\end{aligned}
$$

\section{Applicability}

This section is intended to show through some examples the applicability of our results. Then, programs from various well-known collections are analyzed.

\subsection{Examples}

It is worth noticing that, since the definition of input-consuming derivation is independent from the textual order of the atoms in the clause bodies, the results we have provided (Theorems 20, 24 and 34) hold also in the case that programs and queries are permutation nicely- (or simply-) moded [SHK98], that is programs and queries which would be nicely- (or simply-) moded after a permutation of the atoms in the bodies. Therefore, for instance, we can apply Theorems 20 and 24 to the program FLATTEN as it is presented in [Apt97] (except for the replacement of "\" with ","), i.e.,

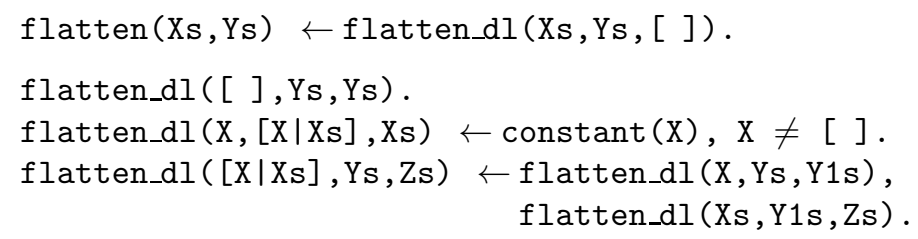

where the atoms in the body of the last clause are permuted with respect to the version of Example 25. 
Let us consider again the program APPEND of the introduction with its natural delay declaration:

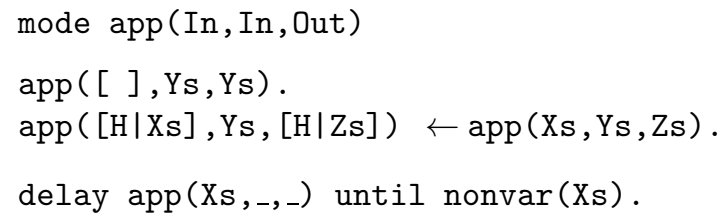

Let $\mathcal{Q}$ be the set of one-atom queries of the form app $(s, t, Z)$ where $s$ and $t$ are any terms and $Z$ is a variable disjoint from $s$ and $t$. Observe that $\mathcal{Q}$ is closed under resolution: Each resolvent in a derivation starting in a query from $\mathcal{Q}$ is still a query from $\mathcal{Q}$. Moreover, because of the presence of the delay declaration, only atoms whose first argument is a non-variable term are allowed to be selected. Thus, selectable atoms have the form $\operatorname{app}(s, t, Z)$ where

(1) $s$ is a non-variable term,

(2) $t$ is any term and $Z$ is a variable disjoint from $s$ and $t$.

Any derivation of APPEND starting in a query of $\mathcal{Q}$ is similar to an inputconsuming one. This follows from the fact that for any selectable atom $A$ and clause's head $H$, there exists a mgu $\theta$ which does not affect the input arguments of $A$. In fact, let $A$ be a selectable atom of $\mathcal{Q}$. If $A$ unifies with the head of the first clause then, by (1), $s$ is the empty list [ ] and $\theta=$ $m g u(A, H)=\{\mathrm{Ys} / t, Z / t\}$. Otherwise, If $A$ unifies with the head of the second clause then, by (1), $s$ is a term of the form $\left[s_{1} \mid s_{2}\right]$ and $\theta=m g u(A, H)=$ $\left\{\mathrm{H} / s_{1}, \mathrm{Xs} / s_{2}, \mathrm{Ys} / t, Z /\left[s_{1} \mid \mathrm{Zs}\right]\right\}$. By (2) it follows that, in both cases, $s \theta=s$ and $t \theta=t$, i.e., $\theta$ does not affect the input arguments of $A$.

Moreover, it is easy to check that APPEND is quasi recurrent wrt. the moded level mapping depicted in Example 17. Since it is nicely-moded, by applying Theorem 20 it follows that it is input terminating. By the arguments above, we can conclude that all the derivations of APPEND in presence of the delay declaration $d_{1}$ and starting in a (permutation) nicely-moded query are finite. Hence, in particular, we can state that all the derivations of APPEND starting in the query $Q_{1}$ of the introduction, which is not nicely-moded but it is permutation nicely-moded, are finite.

\subsection{Benchmarks}

In order to assess the applicability of our results, we have looked into four collections of logic programs, and we have checked those programs against the three classes of programs: (permutation) nicely-moded, input terminating and quasi recurrent programs. The results are reported in Tables 1 to 4 . These tables clearly show that our results apply to the large majority of the programs considered.

In Table 1 the programs from Apt's collection are considered, see Apt97. The programs from the DPPD's collection, maintained by Leuschel and available 
at the URL: http://dsse.ecs.soton.ac.uk/ mal/systems/dppd.html, are referred to in Table 2. Table 3 considers various programs from Lindenstrauss's collection (see the URL: http://www.cs.huji.ac.il/ naomil). Finally, in Table 4 one finds the (almost complete) list of programs by F. Bueno, M. Garcia de la Banda and M. Hermenegildo that can be found at the URL: http://www.clip.dia.fi.upm.es.

For each program we specify the name and the modes of the main procedure. Then we report whether or not the program is (permutation) nicelymoded (NM), input terminating (IT), and quasi recurrent (QR). Notice that for programs which are not input terminating, because of Theorem 20, it does not make sense to check whether or not they are quasi recurrent. For this reason, we leave blank the cells in the column QR corresponding to non-input terminating programs.

Finally, Table 5 reports the list of programs from previous tables which have been found to be input terminating but not quasi recurrent. For these programs, the notion of quasi recurrency does not provide an exact characterization of input termination. In particular, Theorem 34 does not apply. In order to understand which of the hypothesis of the theorem does not hold, we report in Table 5 whether or not these programs are simply-moded (SM) and inputrecursive (IR).

\section{Conclusion and Related Works}

In this paper we studied the properties of input-consuming derivations of nicelymoded programs.

This study is motivated by the widespread use of programs using dynamic scheduling controlled by delay declarations. In fact, as we have motivated in Section 3.2, we believe that in most practical programs employing delay declarations these constructs are used for guaranteeing that the derivation steps are input-consuming.

In the first place, we showed that for nicely-moded programs a weak version of the well-known switching lemma holds: If, given a query $(\mathbf{A}, B, \mathbf{C}, D, \mathbf{E})$, $D$ is selected before $B$ in an input-consuming derivation, then the two resolution steps can be interchanged while maintaining that the derivation is inputconsuming.

Secondly, we presented a method for proving termination of programs and queries which are (permutation) nicely-moded. We also showed a result characterizing a class of input terminating programs.

In the literature, the paper most related to the present one is certainly Sma99b. Our results strictly generalize those in Sma99b in the fact that we drop the condition that programs and queries have to be well-moded. This is particularly important in the formulation of the queries. For instance, in the program FLATTEN of Example 25, our results show that every input-consuming derivation starting in a query of the form $f \operatorname{latten}(t, s)$ terminates provided that $t$ is linear and disjoint from $s$, while the results of Sma99b apply only if $t$ is a ground term. Note that well-moded queries (in well-moded programs) never 
terminate by deadlock, since the leftmost atom of each resolvent is ground in its input positions and hence selectable. This does not hold for nicely-moded queries which might deadlock. Our method allows us thus to cope also with this more difficult situation: For instance we can prove that all derivations of APPEND starting in $\operatorname{app}(\mathrm{X}, \mathrm{Y}, \mathrm{Z})$ are terminating. In practice the result of [Sma99b] identify a class of programs and queries which is both terminating and deadlock free. While deadlock is clearly an undesirable situation, there are various reasons why one might want to prove termination independently from the absence of deadlock: In the first place, one might want to prove absence of deadlock using a different tool than by employing well-moded programs. Secondly, in some situations absence of deadlock might be difficult or impossible to prove, like in a modular context in which the code of some module is not known, hence not analyzable: consider for instance the query generator_1(X1s), generator_2(X2s), append $(\mathrm{X} 1 \mathrm{~s}, \mathrm{X} 2 \mathrm{~s}, \mathrm{Zs})$., where the generators are defined in different modules; our results allow us to demonstrate that if the generators terminate, then the above query terminates. On the other hand, one cannot determine whether it is deadlock free unless one has a more precise specification of the generators. Thirdly, it is well-known that one of the goals of dynamic scheduling is precisely enforcing termination; in this respect a deadlock can be regarded as the situation in which "all else failed". Our system allows us to check how effective dynamic scheduling is in enforcing termination.

Concluding our comparison with Sma99], for the class of (permutation) simply-moded and input-recursive programs, we provide an exact characterization of input termination. A similar result is not present in SSma99b].

Apt and Luitjes [AL95] have also tackled the problem of the termination of programs in presence of dynamic scheduling. The techniques employed in it are based on determinacy checks and the presence of successful derivations, thus are completely different from ours. It is nevertheless worth mentioning that AL95 reports a special ad-hoc theorem, in order to prove that, if $u$ is linear and disjoint from $s$ then the query app $(s, t, u)$ terminates. This is reported in order to show the difficulties one encounters in proving termination in presence of dynamic scheduling. Now, under the further (mild) additional condition that $u$ be disjoint from $t$, the termination of $\operatorname{app}(s, t, u)$ is a direct consequence of our main result.

Another related paper is the one by Marchiori and Teusink MT95. However, Marchiori and Teusink make a strong restriction on the selection rule, which has to be local; this restriction actually forbids any form of coroutining. Moreover, MT95. allows only safe delay declarations; we do not report here the definition of safe delay declaration, we just say that it is rather restrictive: For instance, the delay declaration we have used for APPEND is not safe (a safe one would be delay $\operatorname{app}\left(\mathrm{X},{ }_{-},-\right.$) until list $\left.(\mathrm{X})\right)$. Actually, their requirements go beyond ensuring that derivations are input-consuming.

Applicability and effectiveness of our results have been demonstrated by matching our main definitions against the programs of four public program lists. These benchmarks showed that most of the considered programs are nicelymoded (for a suitable mode) and quasi recurrent (wrt. a suitable level mapping). 


\section{References}

[AB91] K. R. Apt and M. Bezem. Acyclic programs. New Generation Computing, 9(3\&4):335-363, 1991.

[AL95] K. R. Apt and I. Luitjes. Verification of logic programs with delay declarations. In A. Borzyszkowski and S. Sokolowski, editors, Proceedings of the Fourth International Conference on Algebraic Methodology and Software Technology, (AMAST'95), Lecture Notes in Computer Science, Berlin, 1995. Springer-Verlag.

[AM94] K. R. Apt and E. Marchiori. Reasoning about Prolog programs: from Modes through Types to Assertions. Formal Aspects of Computing, 6(6A):743-765, 1994.

[AP93] K. R. Apt and D. Pedreschi. Reasoning about termination of pure Prolog programs. Information and Computation, 106(1):109-157, 1993.

[AP94a] K. R. Apt and D. Pedreschi. Modular termination proofs for logic and pure Prolog programs. In G. Levi, editor, Advances in Logic Programming Theory, pages 183-229. Oxford University Press, 1994.

[AP94b] K. R. Apt and A. Pellegrini. On the occur-check free Prolog programs. ACM Toplas, 16(3):687-726, 1994.

[Apt90] K. R. Apt. Introduction to Logic Programming. In J. van Leeuwen, editor, Handbook of Theoretical Computer Science, volume B: Formal Models and Semantics, pages 495-574. Elsevier, Amsterdam and The MIT Press, Cambridge, 1990.

[Apt97] K. R. Apt. From Logic Programming to Prolog. Prentice Hall, 1997.

[Bez93] M. Bezem. Strong termination of logic programs. Journal of Logic Programming, 15(1\&2):79-97, 1993.

[Cav89] L. Cavedon. Continuity, consistency and completeness properties for logic programs. In G. Levi and M. Martelli, editors, 6 International Conference on Logic Programming, pages 571-584. MIT press, 1989.

[Cav91] L. Cavedon. Acyclic programs and the completeness of SLDNFresolution. Theoretical Computer Science, 86:81-92, 1991.

[DD94] D. De Schreye and S. Decorte. Termination of logic programs: the never-ending story. Journal of Logic Programming, 19-20:199-260, 1994.

[EBC99] S. Etalle, A. Bossi, and N. Cocco. Termination of well-moded programs. Journal of Logic Programming, 38(2):243-257, 1999. 
[FGMP97] M. Falaschi, M. Gabbrielli, K. Marriot, and C. Palamidessi. Constraint logic programming with dynamic scheduling: a semantics based on closure operators. Information and Computation, 137(1):41-67, 1997.

[HL94] P. M. Hill and J. W. Lloyd. The Gödel programming language. The MIT Press, 1994.

[Llo87] J. W. Lloyd. Foundations of Logic Programming. Symbolic Computation - Artificial Intelligence. Springer-Verlag, Berlin, 1987. Second edition.

[Mar97] K. Marriot. Algebraic and logical semantics for clp languages with dynamic scheduling. Journal of Logic Programming, 32(1):71-84, 1997.

[MT95] E. Marchiori and F. Teusink. Proving termination of logic programs with delay declarations. In J. Lloyd, editor, Proc. Twelfth International Logic Programming Symposium. MIT Press, 1995.

[Nai88] L. Naish. Parallelizing NU-Prolog. In Kenneth A. Bowen and Robert A. Kowalski, editors, Proceedings of the Fifth International Conference/Symposium on Logic Programming, pages 1546-1564, Seattle, Washington, August 1988.

[Nai92] L. Naish. Coroutining and the construction of terminating logic programs. Technical Report 92/5, Department of Computer Science, University of Melbourne, 1992.

[SHC96] Z. Somogyi, F. Henderson, and T. Conway. The execution algorithm of Mercury, an efficient purely declarative logic programming language. Journal of Logic Programming, 29(1-3):17-64, 1996.

[SHK98] J.-G. Smaus, P. M. Hill, and A. M. King. Termination of logic programs with block declarations running in several modes. In C. Palamidessi, editor, Proceedings of the 10th Symposium on Programming Language Implementations and Logic Programming, LNCS. Springer-Verlag, 1998.

[SIC97] Intelligent Systems Laboratory, Swedish Institute of Computer Science, PO Box 1263, S-164 29 Kista, Sweden. SICStus Prolog User's Manual, 1997. http://www.sics.se/isl/sicstus/sicstus_toc.html.

[Sma99a] J.-G. Smaus. Modes and Types in Logic Programming. PhD thesis, University of Kent at Canterbury, October 1999. Draft available from www.cs.ukc.ac.uk/people/staff/jgs5/thesis.ps. 
[Sma99b] J.-G. Smaus. Proving termination of input-consuming logic programs. In D. De Schreye, editor, Proceedings of the 16th International Conference on Logic Programming, pages 335-349. MIT Press, 1999.

[Ued88] K. Ueda. Guarded Horn Clauses, a parallel logic programming language with the concept of a guard. In M. Nivat and K. Fuchi, editors, Programming of Future Generation Computers, pages 441-456. North Holland, Amsterdam, 1988.

[UM94] K. Ueda and M. Morita. Moded flat GHC and its message-oriented implementation technique. New Generation Computing, 13(1):3-43, 1994.

[WNS97] M. G. Wallace, S. Novello, and J. Schimpf. Eclipse : A platform for constraint logic programming. ICL Systems Journal, 12(1), 1997. 
Table 1: Programs from Apt's Collection

\begin{tabular}{|c|c|c|c|c|c|c|c|}
\hline & NM & IT & 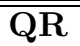 & & $\overline{\mathrm{NM}}$ & IT & $\overline{\text { QR }}$ \\
\hline $\operatorname{app}\left(\operatorname{In},_{-,-}\right)$ & yes & yes & yes & ordered(In) & yes & yes & yes \\
\hline $\operatorname{app}\left({ }_{-,}, \operatorname{In}\right)$ & yes & yes & yes & overlap(_,In) & yes & yes & yes \\
\hline $\operatorname{app}($ Out,In,Out) & yes & no & & overlap(In,Out) & yes & no & \\
\hline append3(In,In,In,Out) & yes & yes & yes & $\operatorname{perm}(,$, In $)$ & yes & yes & yes \\
\hline color_map(In,Out) & yes & no & & perm(In,Out) & yes & no & \\
\hline color_map(Out,In) & yes & no & & qsort(In,_) & yes & yes & no \\
\hline color_map(In,In) & yes & yes & yes & qsort(Out,In) & yes & no & \\
\hline dcsolve(In,_) & yes & no & & reverse $(\operatorname{In},-)$ & yes & yes & yes \\
\hline even(In) & yes & yes & yes & reverse(Out,In) & yes & no & \\
\hline fold(In,In,Out) & yes & yes & yes & $\operatorname{select}\left(\_, \operatorname{In},-\right)$ & yes & yes & yes \\
\hline list(In) & yes & yes & yes & $\operatorname{select}(,,,$, In $)$ & yes & yes & yes \\
\hline $\operatorname{lte}(\operatorname{In},-)$ & yes & yes & yes & select(In,Out,Out) & yes & no & \\
\hline $\operatorname{lte}($ _,In) & yes & yes & yes & subset(In,In) & yes & yes & yes \\
\hline $\operatorname{map}(\operatorname{In},-)$ & yes & yes & yes & subset (In,Out) & yes & no & \\
\hline $\operatorname{map}\left(\_,\right.$In $)$ & yes & yes & yes & subset (Out,In) & yes & no & \\
\hline member(_,In) & yes & yes & yes & $\operatorname{sum}\left(\_,\right.$In,_- $)$ & yes & yes & yes \\
\hline member(In,Out) & yes & no & & $\operatorname{sum}\left({ }_{-,}, \operatorname{In}\right)$ & yes & yes & yes \\
\hline mergesort(In,_) & yes & yes & no & sum(In,Out,Out) & yes & no & \\
\hline mergesort(Out,In) & yes & no & & type(In,In,Out) & no & yes & no \\
\hline mergesort_variant $_{-,-,}$, In $)$ & yes & yes & yes & type(In,Out,Out) & no & no & \\
\hline
\end{tabular}

Table 2: Programs from DPPD's Collection

\begin{tabular}{llll||lccc}
\hline \hline & NM & IT & QR & & NM & IT & QR \\
\hline applast(In,In,Out) & yes & yes & yes & match_app(In,Out) & yes & no & \\
\hline applast(Out,_,_) & yes & no & & max_lenth(In,Out,Out) & yes & yes & yes \\
\hline applast(_,Out,_) & yes & no & & memo_solve(In,Out) & yes & yes & no \\
\hline contains(_,In) & yes & yes & yes & power(In,In,In,Out) & yes & yes & yes \\
\hline contains(In,Out) & yes & no & & prune(In,_) & yes & yes & yes \\
\hline depth(In,In) & yes & yes & yes & prune(_,In) & yes & yes & yes \\
\hline depth(In,Out) & yes & yes & no & relative (In,_) & yes & no & \\
\hline depth(Out,In) & yes & no & & relative(_,In) & yes & no & \\
\hline duplicate(In,Out) & yes & yes & yes & rev_acc(In,In,Out) & yes & yes & yes \\
\hline duplicate(Out,In) & yes & yes & yes & rotate(In,_) & yes & yes & yes \\
\hline flipflip(In,Out) & yes & yes & yes & rotate(_,In) & yes & yes & yes \\
\hline flipflip(Out,In) & yes & yes & yes & solve(_,_,_) & yes & no & \\
\hline generate(In,In,Out) & yes & no & & ssupply(In,In,Out) & yes & yes & yes \\
\hline liftsolve(In,Out) & yes & no & & trace(In,In,Out) & yes & yes & yes \\
\hline liftsolve(Out,In) & yes & no & & transpose(_,In) & yes & yes & yes \\
\hline liftsolve(In,In) & yes & yes & yes & transpose(In,Out) & yes & no & \\
\hline match_app(_,In) & yes & yes & yes & unify(In,In,Out) & yes & no & \\
\hline \hline
\end{tabular}


Table 3: Programs from Lindenstrauss's Collection

\begin{tabular}{|c|c|c|c|c|c|c|c|}
\hline & NM & $\overline{\mathrm{IT}}$ & $\overline{\mathrm{QR}}$ & & $\overline{\mathrm{NM}}$ & $\overline{\mathrm{IT}}$ & QR \\
\hline $\operatorname{ack}(\operatorname{In}, \operatorname{In},-)$ & yes & yes & no & least(In,_) & yes & yes & yes \\
\hline concatenate(In,,-_) & yes & yes & yes & least(_,In) & yes & yes & yes \\
\hline concatenate $\left(,_{-}\right.$, In $)$ & yes & yes & yes & normal_form(In,_) & yes & no & \\
\hline concatenate (_,In,_) & yes & no & & normal_form(_,In) & yes & no & \\
\hline descendant(In,_) & yes & no & & queens(_,Out) & yes & yes & no \\
\hline descendant(_,In) & yes & no & & queens(_,,In) & yes & yes & yes \\
\hline $\operatorname{deep}\left(\operatorname{In},,_{-}\right)$ & yes & yes & yes & $\operatorname{poss}(\operatorname{In})$ & yes & yes & yes \\
\hline deep(Out,_) & yes & no & & poss(Out) & yes & no & \\
\hline credit $\left(\operatorname{In},,_{-}\right)$ & yes & yes & yes & rewrite $(\operatorname{In},-)$ & yes & yes & yes \\
\hline $\operatorname{credit}\left(\_,\right.$In $)$ & yes & yes & yes & rewrite(_,In) & yes & yes & yes \\
\hline holds(_,Out) & yes & no & & transform $\left({ }_{-,-,}\right.$, Out $)$ & yes & no & \\
\hline $\operatorname{holds}(,$, In $)$ & yes & yes & yes & $\operatorname{transform}(,,-,-$, In $)$ & yes & yes & yes \\
\hline huffman(In,_) & yes & yes & no & twoleast(In,_) & yes & yes & yes \\
\hline huffman(_,In) & yes & no & & twoleast(_,In) & yes & yes & yes \\
\hline
\end{tabular}

Table 4: Programs from Hermenegildo's Collection

\begin{tabular}{llccc}
\hline \hline & & NM & IT & QR \\
\hline aiakl.pl & init_vars(In,In,Out,Out) & yes & yes & yes \\
\hline ann.pl & analyze_all(In,Out) & yes & yes & yes \\
\hline bid.pl & bid(In,Out,Out,Out) & yes & yes & yes \\
\hline boyer.pl & tautology(In) & yes & no & \\
\hline browse.pl & investigate(In,Out) & yes & yes & yes \\
\hline fib.pl & fib(In,Out) & yes & no & \\
\hline fib_add.pl & fib(In,Out) & yes & yes & yes \\
\hline hanoiapp.pl & shanoi(In,In,In,In,Out) & yes & no & \\
\hline hanoiapp_suc.pl & shanoi(In,In,In,In,Out) & yes & yes & yes \\
\hline mmatrix.pl & mmultiply(In,In,Out) & yes & yes & yes \\
\hline occur.pl & occurall(In,In,Out) & yes & yes & yes \\
\hline peephole.pl & peephole_opt(In,Out) & yes & yes & yes \\
\hline progeom.pl & pds(In,Out) & yes & yes & yes \\
\hline rdtok.pl & read_tokens(In,Out) & yes & no & \\
\hline read.pl & parse(In,Out) & yes & no & \\
\hline serialize.pl & serialize(In,Out) & yes & yes & no \\
\hline tak.pl & tak(In,In,In,Out) & yes & no & \\
\hline tictactoe.pl & play(In) & yes & no & \\
\hline warplan.pl & plans(In,In) & yes & no & \\
\hline \hline
\end{tabular}


Table 5: Input terminatining but non-quasi recurrent Programs

\begin{tabular}{lcc}
\hline \hline & SM & IR \\
\hline mergesort(In,-) & yes & no \\
\hline qsort(In,-) & yes & no \\
\hline type(In,In,Out) & no & no \\
\hline depth(In,Out) & yes & no \\
\hline memo_solve(In,Out) & no & no \\
\hline ack(In,In,-) & yes & no \\
\hline huffman(In,-) & no & no \\
\hline queens(_,Out) & no & no \\
\hline serialize(In,Out) & no & no \\
\hline \hline
\end{tabular}

